\title{
Spherocylindrical Microplane Constitutive Model for Shale and Other Anisotropic Rocks
}

\author{
Cunbao Li ${ }^{\mathrm{a}, 1}$, Ferhun C. Caner ${ }^{\mathrm{b}, 2}$, Viet T. Chau ${ }^{\mathrm{a}, 3}$, Zdeněk P. Bažant ${ }^{\mathrm{a}, 4, *}$ \\ ${ }^{a}$ Northwestern University, 2145 Sheridan Road, CEE, Evanston, IL 60208, United States \\ ${ }^{b}$ Institute of Energy Technologies, Technical University of Catalonia, Av. Diagonal 647, 08028 Barcelona, \\ Spain
}

\begin{abstract}
Constitutive equations for inelastic behavior of anisotropic materials have been a challenge for decades. Presented is a new spherocylindrical microplane constitutive model that meets this challenge for the inelastic fracturing behavior of orthotropic materials, and particularly the shale, which is transversely isotropic and is important for hydraulic fracturing (aka fracking) as well as many geotechnical structures. The basic idea is to couple a cylindrical microplane system to the classical spherical microplane system. Each system is subjected to the same strain tensor while their stress tensors are superposed. The spherical phase is similar to the previous microplane models for concrete and isotropic rock. The integration of stresses over spherical microplanes of all spatial orientations relies on the previously developed optimal Gaussian integration over a spherical surface. The cylindrical phase, which is what creates the transverse isotropy, involves only microplanes that are normal to plane of isotropy, or the bedding layers, and enhance the stiffness and strength in that plane. Unlike all the microplane models except the spectral one, the present one can reproduce all the five independent elastic constants of transversely isotropic shales. Vice versa, from these constants, one can easily calculate all the microplane elastic moduli, which are all positive if the elastic in-to-out-of plane moduli ratio is not too big (usually less than 3.75, which applies to all shales). Oriented micro-crack openings, frictional micro-slips and bedding plane behavior can be modeled more intuitively than with the spectral approach. Data fitting shows that the microplane resistance depends on the angle with the bedding layers non-monotonically, and compressive resistance reaches a minimum at $60^{\circ}$. A robust algorithm for explicit step-by-step structural analysis is formulated. Like all microplane models, there are many material parameters, but they can be identified sequentially. Finally, comparisons with extensive test data for shale validate the model.
\end{abstract}

Keywords: constitutive models, inelastic behavior, material damage, orthotropic materials, transverse isotropy, geotechnical structures, hydraulic fracturing (fracking), test data fitting, computational mechanics

\footnotetext{
${ }^{*}$ Corresponding author. Tel.: +1 847491 4025; Fax: +1 8474914011.

Email address: z-bazant@northwestern.edu (Zdeněk P. Bažant )

${ }^{1}$ Graduate candidate, Key Laboratory of Energy Engineering Safety and Disaster Mechanics Ministry of Education (Sichuan University) and College of Architecture and Environment, Sichuan University; Research Fellow, Northwestern University.

${ }^{2}$ Associate Professor at the Institute of Energy Technologies, Technical University of Catalonia.

${ }^{3}$ Graduate Research Assistant, Northwestern University.

${ }^{4}$ McCormick Institute Professor and W.P. Murphy Professor of Civil and Mechanical Engineering and Materials Science.
} 


\section{Introduction}

Despite the astonishing recent success of hydraulic fracturing technology, aka fracking (Clark et al., 2013), the mechanics of shale fracturing is still poorly understood and major gaps of knowledge remain (Bažant et al., 2014; Xie et al., 2016). This may partly explain why only 5\% to $15 \%$ of the gas contained in the shale strata is currently getting extracted. Increasing this percentage requires tackling a number of problems. One of them is the problem of material constitutive model for fracturing damage and frictional slip in shale.

Mainly because of the pronounced anisotropy of shale, no realistic constitutive model exists at present. It is needed to design and control the fracking for gas or oil, as well as deep underground sequestration of $\mathrm{CO}_{2}$, fracking water and other toxic fluids, and for radioactive waste disposal. No less it is needed for safety assessments of tunnels and underground caverns, foundations of tall building or bridges, and all kinds of geotechnical excavations in shale.

The purpose of this study is to develop a new version of microplane constitutive model called spherocylindrical (whose basic idea was originally suggested in Bažant's recent conference article (Bažant, 2017). This model can handle progressive softening damage in presence of orthotropy or transverse isotropy, two special cases of anisotropy. It does so by coupling a cylindrical microplane system with the classical spherical microplane system.

\section{Overview of previous studies}

There exist many reports on material tests of rocks with innate transverse isotropy, a special case of anisotropy. They are particularly extensive for shale, for which they include the uniaxial compression tests (Kim et al., 2012), Brazilian split-cylinder tests (Mokhtari et al., 2014; Vervoort et al., 2014), direct shear tests (Heng et al., 2015), triaxial compression tests (Niandou et al., 1997; Masri et al., 2014; Mohamadi and Wan, 2016), scratch tests (Akono, 2016), and uniaxial and triaxial creep tests (Sone, 2012; Chang and Zoback, 2009). These test results demonstrate that the stiffness and strength of shale depends strongly on the loading direction with respect to the bedding layers, which are the planes of isotropic (rotational) symmetry.

Unfortunately, most published data are limited to tests where the bedding layers are either perpendicular or parallel to the loading direction. Not surprisingly, the parallel direction is what gives the maximum compressive strength. Interestingly, though, compression tests of varying directions reveal that the minimum compressive strength does not occur for the parallel and orthogonal directions. Rather, it occurs when the angle of the principal compressive stress direction with the bedding layers is $30^{\circ}$ to $60^{\circ}$ (Fig. 1). The uniaxial tensile strength is found to increase with the bedding layer inclination. The main mechanisms of failure appears to be the extension and sliding of along bedding planes, the splitting, and the shear band slip in shale matrix.

The failure criteria for anisotropic geomaterials have also been studied. Duveau et al. (1998) distinguished anisotropic failure criteria into three kinds: the mathematical continuum models (Tsai and Wu, 1971; Hill, 1998), empirical continuum models (Ramamurthy et al., 1988) and discontinuous weakness plane models (Jaeger, 1960). Some researchers (Gao et al., 2010; Lee and Pietruszczak, 2015) also formulated anisotropic failure criteria by combining the isotropic ones with the fabric tensor. Often the inherent material anisotropy was confused with stressinduced incremental anisotropy, which is naturally exhibited even by isotropic materials. A pervasive limitation has been that only a very limited part of the available test data has been used for experimental validation of these models. 


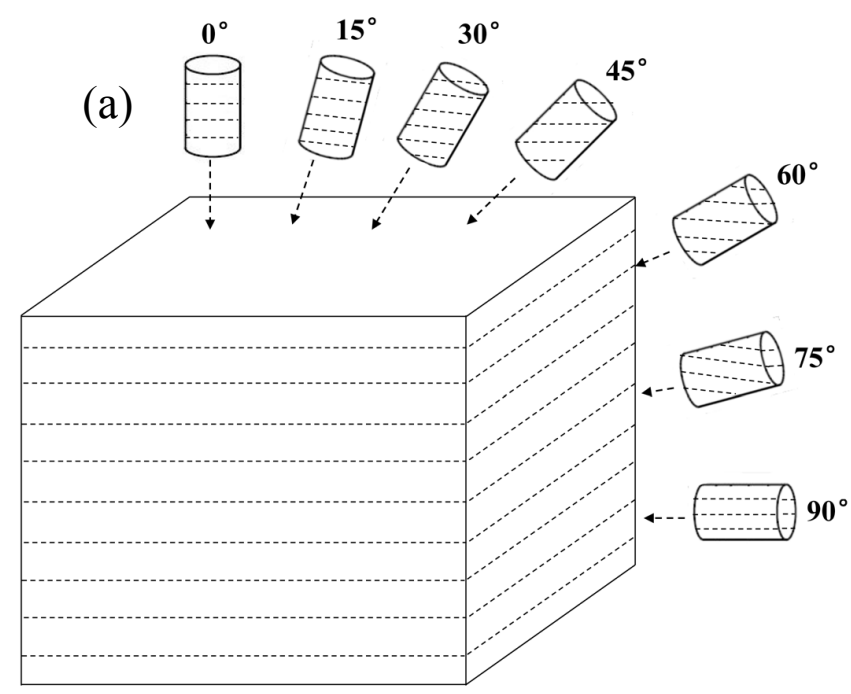

(b)

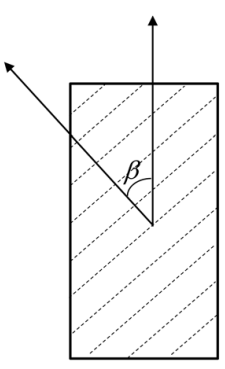

Figure 1: (a) Directional coring diagram of specimens with different bedding layer orientations; (b) definition of the bedding layer orientation.

A major problem is the widespread use of stress-based failure criteria, or yield criteria of plasticity. Such criteria ignore the inevitable size effect due to damage localization, and if they include softening damage they lead to spurious mesh sensitivity. This is unrealistic for most rocks, including shale.

The finite element analysis necessitates formulating complete stress-strain relations, with progressive softening damage included (in a nonlocal sense, of course, with a localization limiter). Attempts for such stress-strain relations have been formulated tensorially, often in the framework of irreversible thermodynamics with internal variables (of non-specific orientations, though) (Chen et al., 2010; Levasseur et al., 2015; Pietruszczak et al., 2002; Jin et al., 2017b).

Regrettably, the use of stress and strain tensors with orthotropic invariants has made it virtually impossible to capture the damage orientation. This has been a major drawback. For example, dependence of the second invariant of the stress deviator, $J_{2}$, on the first stress invariant, $I_{1}$, is widely considered to characterize internal friction. In reality, though, the frictional slips, as well as damage due to microcracking, occur only along planes of certain distinct orientations, which cannot be captured tensorially.

\section{Microplane modeling philosophy and gradual progress}

The oriented character of damage can be captured by the microplane modeling concept. Its history is long. It began in 1938 with G.I. Taylor's (Taylor, 1938) idea to formulate the constitutive relation in terms of the stress and strain vectors acting on a generic plane within the material. Initially, the stress vector was assumed to be the projection of the stress tensor on this plane. This was a static constraint, which led to Batdorf and Budianski's slip theory of plasticity (Batdorf and Budiansky, 1949) and culminated with the recent success of the Taylor models for plastic hardening of polycrystals (Asaro and Rice, 1977; McDowell, 2008, e.g.).

In 1984 (Bažant, 1984; Bažant and Gambarova, 1984), it was shown that for quasibrittle materials, which exhibit softening damage, the static constraint must be replaced, for reasons of stability (as well as explicitness of computations), by a kinematic constraint. In that constraint, the strain (rather than stress) vector on a generic plane in the material microstructure (for which the term 'microplane' was coined) is a projection of the continuum strain tensor, while the stress 
vector is calculated from the strain vector by the microplane constitutive law. Furthermore, it was shown that, in the case of softening damage, the simple superposition of the plastic strain vectors used in Taylor models must be replaced by virtual work (variational) equivalence between the stress tensor and the microplane stress vectors, and that the elasticity, too, must be included in the microplane constitutive law rather than on the tensorial macro-level. For isotropic randomly heterogeneous materials, the microplanes may be regarded as the tangent planes of a unit sphere surrounding every material point (Fig. 2(a)). A crucial advantage is that the orientation of the basic damage mechanisms, such as frictional slip or crack opening, can be intuitively captured on the microplanes.

The microplane model has been progressively improved for concrete through versions M1, M2, ...M7 (Bažant and Oh, 1985; Bažant and Prat, 1988; Bažant et al., 1996, 2000b; Bažant and Caner, 2005; Caner and Bažant, 2011, 2013) and has recently been widely applied in finite element analysis (Bažant et al., 2000a; Caner and Bažant, 2014, e.g.). It was also adapted to other isotropic randomly heterogeneous quasibrittle materials, particularly rocks (Bažant and Zi, 2003; Chen and Bažant, 2014) and clays (Bažant and Kim, 1986; Bažant and Prat, 1987). The thermodynamic restrictions of microplane model M7 have been elucidated in (Bažant and Caner, 2014). The microplane model for concrete is now embedded in various commercial softwares (e.g., ATENA, DIANA, SBETA), open-source codes (e.g., OOFEM) and large wavecodes (e.g., EPIC, PRONTO, MARS). For isotropic jointed rock mass, it is featured as a user subroutine in ANSYS (Bažant et al., 2015).

Initially, the microplane model was too demanding computationally. But the inexorable rise of computer power removed this obstacle after the advent of the 21st century. While, in comparison to a simple tensorial constitutive law (such as Drucker-Prager), the run time of a microplane finite element program may be 10-times longer for a system of 10 elements, it is only a few percent longer for 10 million elements. The reason is that, for explicit integration, the computer run time increases linearly with the demand of the constitutive law but faster than linearly with the number of degrees of freedom (DOF), though slower than quadratically, which would apply to implicit integration.

Like for all kinds of constitutive laws, material anisotropy (or orthotropy) has proven to be a major challenge, especially for damage modeling. The simplest way to achieve anisotropy is to include orientation dependent weights for the microplanes. But this worked only for the mild transverse isotropy of foam cores of sandwich shells (due to elongated pore shape (Brocca et al., $2001)$ ), in which case the ratio of in-plane to out-of-plane elastic moduli, $E_{x x} / E_{y y}$, is about 2. More generally, varying the microplane stiffness and strength parameters as functions of the polar angle has been tried for textile composites as well as shale(Li et al., 2017), but could not fit the test data well. Recently it was rigorously proven (Cusatis et al., 2008; Jin et al., 2017a) that mere microplane weighting cannot capture general orthotropy. A remedy was found in the spectral stiffness microplane model based on the eigenvectors of the orthotropic stiffness matrix. But although the spectral approach to orthotropy is fully general, it is too abstract, allows only limited physical insight while being non-intuitive and unwieldy in fitting extensive material data.

Another type of orthotropic microplane model has been developed specifically for the textile composites, to capture yarn directions and undulation effects. On the subscale, the yarn microplanes normal to the yarn are imagined to be placed at various points of the yarn wave and then shrunken on the continuum macroscale into the material point (Šmilauer et al., 2011). This approach has recently been refined by replacing each solo microplane with a triad of three coupled orthogonal microplanes, one normal to the undulating yarn and two others parallel to it. The latest 'microplane triad' model (Kirane et al., 2015, 2016) has been shown to predict 
closely, from the constituent properties, all the orthotropic elastic constants and fracturing behavior of textile composites. However, this approach to orthotropy, capturing yarn architecture, is not transplantable to shale. A new approach is needed, as presented here.

\section{General framework of spherocylindrical microplane model}

\subsection{Basic configuration and hypotheses}

To represent a fully general transverse orthotropy of shale and other layered materials with damage, a new approach is here introduced - the spherocylindrical microplane model, which combines spherical and cylindrical microplane systems, herein called phases. The microplane configuration is illustrated in Fig. 2, in which the classical three-dimensional (3D) spherical microplane phase is considered to be coupled in parallel with a two-dimensional (2D) cylindrical microplane phase subjected to the same continuum strain tensor $\epsilon_{i j}$ where $i, j=1,2,3=$ Cartesian subscripts. The strain tensors in the spherical and cylindrical microplane phases are assumed to be the same (which is called the kinematic constraint), and their stresses are added according to the partition of unity concept. The stress tensors carried by the spherical and cylindrical microplane phases are assumed to be $\alpha \sigma_{i j}$ and $(1-\alpha) \sigma_{i j}$, respectively, where $\sigma_{i j}$ is the continuum (or macro scale) stress tensor and $\alpha$ is the (empirical) volume fraction of the spherical phase $(<1$, which is a typical simplification in the mechanics of composites (Hahn and Tsai, 1980)). For Longmaxi shale, the empirically optimized value is $\alpha=0.5$.

The spatial orientations of the microplane normals of the spherical phase are based on the optimal Gaussian numerical integration formula for a spherical surface (Bažant and Oh, 1986), the same as in the original microplane model. These orientations cannot be distributed over the sphere uniformly (because a regular polyhedron cannot have more than 10 sides per hemisphere, and more than 10 points are needed for accuracy). Thus the integration points must have nonuniform weights, $w_{\mu}$ (Bažant and Oh, 1986).

For the cylindrical phase, the numerical integration is trivial. Because of the requirement of rotational invariance in the plane of isotropy, the subdivision of the equatorial circle must be uniform (Fig. 2(a)), with equal weights $1 / N_{c}$ regardless the number, $N_{c}$, of points (or cylindrical microplanes).

Here one should realize that integration from $0^{\circ}$ to $360^{\circ}$ is not the same as integration of a function over a line segment (for which Gauss-Legendre formula give the optimal integration). The reason is that the points $0^{\circ}$ and $360^{\circ}$ coincide physically, and that the location of the 0 point on the circle is arbitrary and thus must not affect the integration weights.

Furthermore, because the microplanes on opposite sides of the circle represent the same stress-strain state, it suffices to integrate (or sum) over only a half-circle and then double the result. Again, the fact that the actual integration is carried out over a half circle, i.e., over the interval $\left(0^{\circ}, 180^{\circ}\right)$ is immaterial for the weighting because it merely substitutes for integration over the whole circle (by analogy, note that, for the spherical integral, it would similarly be incorrect to evaluate it numerically as an integral over the rectangular domain of $\theta \in(0, \pi / 2), \varphi \in(0,2 \pi))$.

The basic hypotheses of the present model are as follows:

Hypothesis I. According to many experimental studies (Gautam and Wong, 2006; Waters et al., 2011), the shale can be seen as a transversely isotropic material (hence, there are five independent elastic constants).

Hypothesis II. The spherical and cylindrical microplane phases are connected in parallel. Therefore, the strain in both phases is the same while the total force in the spherocylindrical model is the sum of the forces in both phases (or systems). 
Hypothesis III. In both the spherical and cylindrical phases, the strain vectors on any microplane are the resolved components of the macro-continuum strain tensor. In other words, like in most microplane models, the microplanes are subjected to a kinematic constraint. Such a constraint is necessary to ensure the stability of strain softening and guarantee a robust explicit algorithm (Bažant and Oh, 1985; Caner and Bažant, 2013).
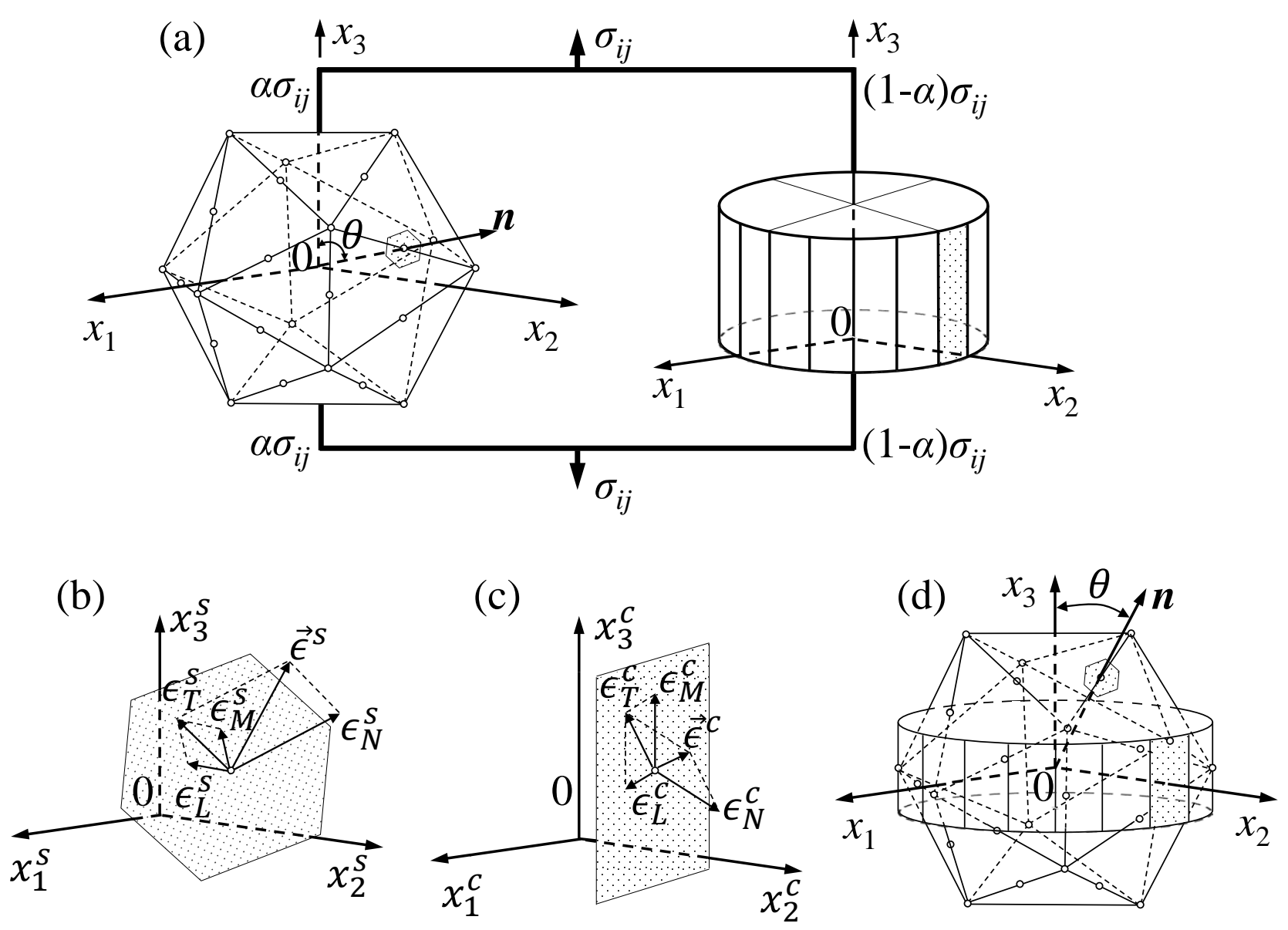

Figure 2: (a) Schematic of the paralleled sphererocylindrical microplane configuration; left: spherical microplane position and microplane dip (or azimuth) angle $\theta$; right: cylindrical microplane configuration; (b) microplane strain components on one microplane of the spherical phase; (c) microplane strain components on one microplane of the cylindrical phase; (d) another schematic of the sphererocylindrical microplane configuration in parallel coupling.

\subsection{Basic relations for kinematically constrained spherocylindrical microplane model}

Some conventions and notations need to be introduced first: $\sigma_{i}^{s}$ and $\epsilon_{i}^{s}(i=N, M, L)$ are the stress and strain vectors on a generic microplane of the spherical phase (Fig. 2(b)), with $N, L$ and $M$ representing the normal direction and two orthogonal directions within one microplane; $\sigma_{i}^{c}$ and $\epsilon_{i}^{c}(i=N, M, L)$ are the stress and strain vectors on a generic microplane of the cylindrical phase (Fig. 2(c)). $x_{i}(i=1,2,3)$ are the global Cartesian coordinates; $x_{i}^{s}$, $i=1,2,3$, are the local Cartesian coordinates of the microplane in the spherical phase; $x_{i}^{c}$, $i=1,2,3$, are the local Cartesian coordinates of the cylindrical microplanes; see Fig.2. In the global coordinate system, $\boldsymbol{n}^{s}$ is the unit normal vector and $\boldsymbol{l}^{s}, \boldsymbol{m}^{s}$ are the two orthogonal 
unit vectors of the spherical microplane; $\boldsymbol{n}^{\boldsymbol{c}}$ is the unit normal vector and $\boldsymbol{l}^{\boldsymbol{c}}, \boldsymbol{m}^{\boldsymbol{c}}$ are the two orthogonal unit vectors of the cylindrical microplane.

Based on the kinematic constraint (hypothesis III), the components of the strain vectors $\vec{\epsilon}_{N}^{s}$ (Fig.2(b)) and $\bar{\epsilon}_{N}^{c}$ (Fig.2(c)) on the spherical and cylindrical microplanes are $\epsilon_{N i}^{s}=\epsilon_{i j} n_{j}^{s}$ and $\epsilon_{N i}^{c}=\epsilon_{i j} n_{j}^{c}$, where $n_{j}^{s}$ and $n_{j}^{c}$ are the components of the unit normal vectors $\boldsymbol{n}^{s}$ and $\boldsymbol{n}^{c}$ defining the microplane orientation (repetition of subscripts implies summation over $i=1,2,3$ ). The components of the strain vectors on the spherical and cylindrical microplanes can be computed as follows:

$$
\begin{aligned}
& \epsilon_{N}^{s}=\epsilon_{i j} N_{i j}^{s}, \quad \epsilon_{L}^{s}=\epsilon_{i j} L_{i j}^{s}, \quad \epsilon_{M}^{s}=\epsilon_{i j} M_{i j}^{s} \\
& \epsilon_{N}^{c}=\epsilon_{i j} N_{i j}^{c}, \quad \epsilon_{L}^{c}=\epsilon_{i j} L_{i j}^{c}, \quad \epsilon_{M}^{s}=\epsilon_{i j} M_{i j}^{c}
\end{aligned}
$$

where

$$
\begin{array}{lll}
N_{i j}^{s}=n_{i}^{s} n_{j}^{s}, & L_{i j}^{s}=\left(l_{i}^{s} n_{j}^{s}+l_{j}^{s} n_{i}^{s}\right) / 2, & M_{i j}^{s}=\left(m_{i}^{s} n_{j}^{s}+m_{j}^{s} n_{i}^{s}\right) / 2 \\
N_{i j}^{c}=n_{i}^{c} n_{j}^{c}, & L_{i j}^{c}=\left(l_{i}^{c} n_{j}^{c}+l_{j}^{c} n_{i}^{c}\right) / 2, & M_{i j}^{c}=\left(m_{i}^{c} n_{j}^{c}+m_{j}^{c} n_{i}^{c}\right) / 2
\end{array}
$$

Here $l_{i}^{s}, m_{i}^{s}, l_{i}^{c}$ and $m_{i}^{c}(i=1,2,3)$ are the components of the unit vector $\boldsymbol{l}^{\boldsymbol{s}}, \boldsymbol{m}^{\boldsymbol{s}}, \boldsymbol{l}^{\boldsymbol{c}}$ and $\boldsymbol{m}^{\boldsymbol{c}}$, respectively. The vector $\boldsymbol{n}^{\boldsymbol{s}}$ represents the orientation of microplane normal and thus the microplane position on the sphere or cylinder. The in-plane vector $\boldsymbol{m}^{\boldsymbol{s}}$ can be chosen arbitrarily while the vector $\boldsymbol{l}^{\boldsymbol{s}}$ is generated as $\boldsymbol{l}^{\boldsymbol{s}}=\boldsymbol{m}^{\boldsymbol{s}} \times \boldsymbol{n}^{\boldsymbol{s}}$. Vector $\boldsymbol{m}^{\boldsymbol{c}}$ is chosen to be normal to both $x_{1}^{c}$ and $x_{2}^{c}$, i.e., $\boldsymbol{m}^{\boldsymbol{c}}=(0,0,1)$, and then $\boldsymbol{l}^{\boldsymbol{c}}=\boldsymbol{m}^{\boldsymbol{c}} \times \boldsymbol{n}^{\boldsymbol{c}}$.

Similar to the classical microplane model for concrete, modeling of the inelastic behavior in compression necessitates in the spherical phase the volumetric-deviatoric split, in which the deviatoric strain on the microplane is defined as

$$
\epsilon_{D}^{s}=\epsilon_{N}^{s}-\epsilon_{V}, \quad \epsilon_{V}=\epsilon_{k k} / 3
$$

where $\epsilon_{V}$ is volumetric (or mean) strain, which is the same for all the spherical microplanes.

In general, it is impossible for both stress and strain vectors on the microplanes to be the projections of the macro-continuum stress and strain tensors. The equilibrium between the microplane stress vectors and the macro-continuum stress tensor is achieved variationally, according to the principle of virtual work and hypothesis II, which is written as

$$
\sigma_{i j} \delta \epsilon_{i j}=\alpha \frac{3}{2 \pi} \int_{\Omega}\left(\sigma_{N}^{s} \delta \epsilon_{N}^{s}+\sigma_{L}^{s} \delta \epsilon_{L}^{s}+\sigma_{M}^{s} \delta \epsilon_{M}^{s}\right) d \Omega+(1-\alpha) \frac{1}{\pi} \int_{S}\left(\sigma_{N}^{c} \delta \epsilon_{N}^{c}+\sigma_{L}^{c} \delta \epsilon_{L}^{c}+\sigma_{M}^{c} \delta \epsilon_{M}^{c}\right) \mathrm{d} S
$$

where $\Omega$ is the surface of a unit hemisphere; $S$ is the surface of a cylinder of unit length and diameter; $\alpha$ is the volumetric fraction of the spherical phase. This equation means that the virtual work of macro-continuum stress must be equal to the sum of the virtual works of microplane stress vectors of both the spherical and cylindrical phases.

Substituting Eq.(1) and Eq.(2) into Eq.(6), one gets the following basic equilibrium relation

$$
\begin{aligned}
\sigma_{i j} & =\alpha \frac{3}{2 \pi} \int_{\Omega}\left(\sigma_{N}^{s} N_{i j}^{s}+\sigma_{L}^{s} L_{i j}^{s}+\sigma_{M}^{s} M_{i j}^{s}\right) d \Omega+(1-\alpha) \frac{1}{\pi} \int_{S}\left(\sigma_{N}^{c} N_{i j}^{c}+\sigma_{L}^{c} L_{i j}^{c}+\sigma_{M}^{c} M_{i j}^{c}\right) \mathrm{d} S \\
& \approx 6 \alpha \sum_{\mu=1}^{N_{m}^{s}} w_{\mu}^{s}\left(\sigma_{N}^{s} N_{i j}^{s}+\sigma_{L}^{s} L_{i j}^{s}+\sigma_{M}^{s} M_{i j}^{s}\right)_{(\mu)}+(1-\alpha) \frac{1}{\pi N_{m}^{c}} \sum_{\mu=1}^{N_{m}^{c}}\left(\sigma_{N}^{c} N_{i j}^{c}+\sigma_{L}^{c} L_{i j}^{c}+\sigma_{M}^{c} M_{i j}^{c}\right)((q))
\end{aligned}
$$

where $N_{m}^{s}$ and $N_{m}^{s}$ are the total numbers of microplanes per hemisphere and per half circle, respectively; $w_{\mu}^{s}$ are the weights in the optimal Gaussian numerical formula for spherical surface (Bažant and Oh, 1986); and subscript $(\mu)$ labels the contribution of the $\mu$-th microplane to 
the macroscopic stress tensor. For accurate integration over the spherical phase, the optimal Gaussian formula for with $N_{m}^{s}=37$ points per hemisphere, derived in (Bažant and Oh, 1986), has been used, and for the cylindrical phase, a uniform subdivision with $N_{m}^{s}=10$.

It might be questioned whether the sphere-cylinder combination might require a change in the integration formula for the hemisphere. It does not because the stresses on the spherical microplanes are independent of the circle subdivision. It has been checked that the present numerical integration of the microplane model reproduces accurately a case for which an exact solution is available - for example, how the elastic stiffness, $C_{\theta}$, in uniaxial compression tests depends on the loading orientation characterized by angle $\theta$; see Fig. 3, which demonstrates that the present numerical integration scheme matches the exact elastic calculations perfectly.

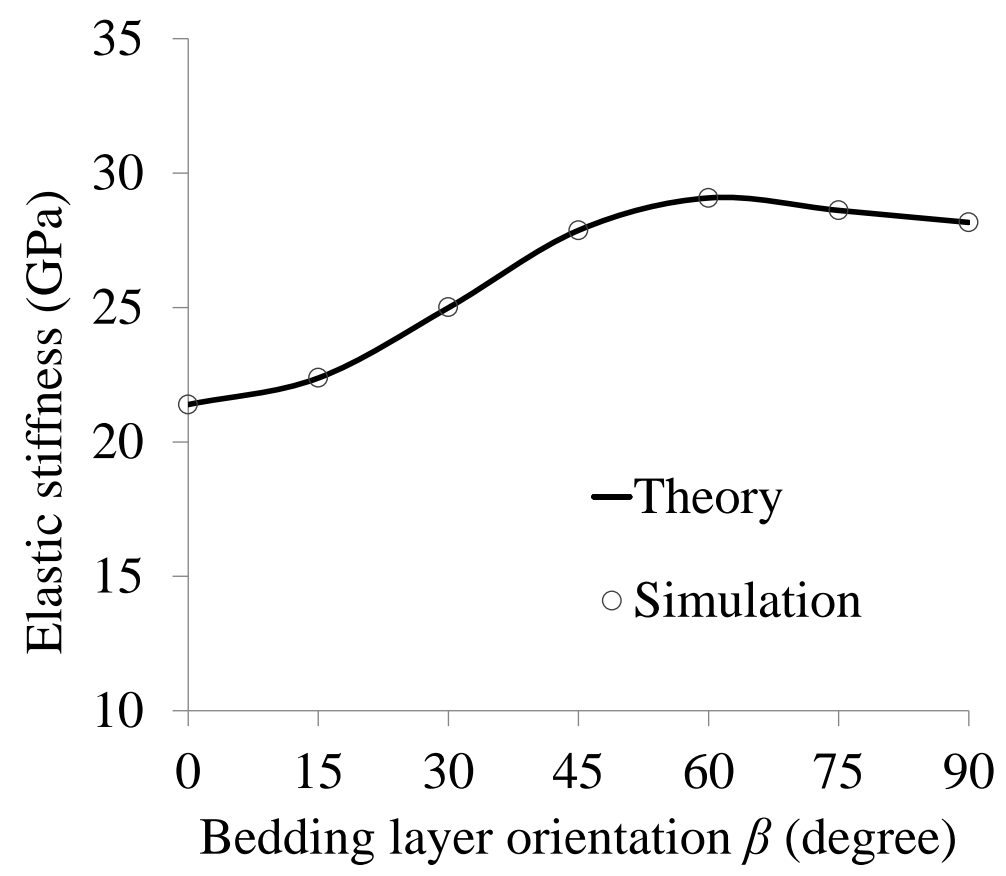

Figure 3: Demonstration of numerical accuracy using a case in which numerical elastic stiffness is compared with theoretical results

\section{Constitutive model on the microplane}

\subsection{Elastic behavior on the microplane}

On the microplane level, two simplifications of elastic behavior have been shown to work:

1. Up to reaching the strength limit, the elastic moduli on the microplanes can be considered constant as long as the loading on the microplane is monotonic. This approximation applies not only to the normal and shear components of the strain vector, but also to the volumetric and deviatoric components. The prepeak nonlinearity and prepeak pathdependence that are seen in material tests are automatically generated by virtue of the fact that, during loading, different microplanes reach their strength limits at different times. Physically, this reflects the gradual formation of microcracks and microslips of different orientations during the loading process.

2. The stress-strain relations for different strain components on the microplanes can be considered decoupled because, as indicated by experience, they are approximately captured by interactions between microplanes of different orientations. 
Consequently,

$$
\begin{aligned}
& \sigma_{V}=E_{V} \epsilon_{V}, \quad \sigma_{N}^{s}=E_{N}^{c} \epsilon_{N}^{s}, \quad \sigma_{D}^{s}=E_{D}^{s} \epsilon_{D}^{s}, \quad \sigma_{M}^{s}=E_{M}^{s} \epsilon_{M}^{s}, \quad \sigma_{L}^{s}=E_{L}^{s} \epsilon_{L}^{s}, \\
& \sigma_{N}^{c}=E_{N}^{c} \epsilon_{N}^{c}, \quad \sigma_{L}^{c}=E_{L}^{c} \epsilon_{L}^{c}, \quad \sigma_{M}^{c}=E_{M}^{c} \epsilon_{M}^{c}
\end{aligned}
$$

where $\sigma_{V}$ and $\sigma_{D}^{s}$ are the microplane normal volumetric stress and deviatoric stress of the spherical phase, respectively; $E_{V}, E_{N}^{s}, E_{D}^{s}, E_{L}^{s}, E_{M}^{s}, E_{N}^{c}, E_{L}^{c}$ and $E_{M}^{c}$ are the elastic parameters on the microplane, considered as independent of microplane orientation.

To obtain the fourth-order stiffness tensor $\bar{C}_{i j k l}$, Eqs.(1), (2), (5), (9) and (10) are substituted into Eq.(6). This yields:

$$
\begin{aligned}
\bar{C}_{i j k l} & =\alpha \frac{3}{2 \pi} \int_{\Omega}\left(E_{D}^{s} N_{i j}^{s} N_{k l}^{s}+\frac{1}{3}\left(E_{V}-E_{D}^{s}\right) N_{i j}^{s} \delta_{k l}+E_{L}^{s} L_{i j}^{s} L_{k l}^{s}+E_{M}^{s} M_{i j}^{s} M_{k l}^{s}\right) \mathrm{d} \Omega \\
& +(1-\alpha) \frac{1}{\pi} \int_{S}\left(E_{N}^{c} N_{i j}^{c} N_{k l}^{c}+E_{L}^{c} L_{i j}^{c} L_{k l}^{c}+E_{M}^{c} M_{i j}^{c} M_{k l}^{c}\right) \mathrm{d} S
\end{aligned}
$$

Since the shear moduli $E_{L}^{s}=E_{M}^{s}$ are applied to the components, $\epsilon_{L}^{s}$ and $\epsilon_{M}^{s}$, of the resultant shear stress vector $\epsilon_{T}^{s}$, both moduli must be equal, i.e., $E_{L}^{s}=E_{M}^{s}=E_{T}^{s}$. Strictly speaking, $E_{T}^{s}$ should be applied to the resultant $E_{T}^{s}=\sqrt{E_{L}^{s^{2}}+E_{M}^{s}}$ (as done in model M2 (Bažant and Prat, 1988)), but this would complicate programming and the gain in accuracy was shown to be insignificant.

In the spherical phase, the ratio $\eta=E_{D}^{s} / E_{L}^{s}$ is ambiguous and may be chosen. For reasons clarified before (Carol et al., 1991), the choice $\eta=1$ is preferable. By comparing the components with the same combinations of matrix subscripts $I J$ on the left and right sides of Eq. (11), one obtains the following expressions for the microplane elastic constants (for a detailed derivation, see Appendix I):

$$
\begin{aligned}
& E_{V}=\left(C_{33}+2 C_{13}\right) / \alpha \\
& E_{L}^{s}=E_{M}^{s}=E_{T}^{s}=\left(C_{33}-C_{13}\right) / \alpha \\
& E_{D}^{s}=\left(C_{33}-C_{13}\right) / \alpha \\
& E_{N}^{c}=2\left(C_{11}-C_{33}+C_{12}-C_{13}\right) /(1-\alpha) \\
& E_{L}^{c}=2\left(C_{11}-C_{33}-3 C_{12}+3 C_{13}\right) /(1-\alpha) \\
& E_{M}^{c}=4\left(2 C_{44}-C_{33}+C_{13}\right) /(1-\alpha)
\end{aligned}
$$

where $C_{11}, C_{12}, C_{13}, C_{33}$ and $C_{44}$ are the five independent components of the transversely isotropic elastic stiffness matrix $C_{I J}(I, J=1,2, \ldots 6)$. This matrix represents the symmetric fourth-order tensor $\bar{C}_{i j k l}$ in the Voigt notation. As the volumetric fraction of the cylindrical phase is 0 , the spherocylindridal microplane model degenerates into an isotropic microplane model, i.e. Eqs. (12-14) can be used to compute microplane elastic constants of isotropic materials and Eqs. (15-17) are meaningless because of singularity. As known in mechanics of materials (Jones, 1975), the stiffness matrix components can be expressed as

$$
\begin{aligned}
& C_{11}=\left(1-\nu_{13} \nu_{31} /\left(E_{11} E_{33} \Delta\right)\right. \\
& C_{12}=\left(\nu_{12}+\nu_{13} \nu_{31} /\left(E_{11} E_{33} \Delta\right)\right. \\
& C_{13}=\left(\nu_{31}+\nu_{12} \nu_{31} /\left(E_{11} E_{33} \Delta\right)\right. \\
& C_{33}=\left(1-\nu_{12}^{2}\right) /\left(E_{11}^{2} \Delta\right) \\
& C_{44}=G_{31}
\end{aligned}
$$




$$
\text { where } \quad \Delta=\left(1+\nu_{12}\right)\left(1-\nu_{12}-2 \nu_{13} \nu_{31}\right) /\left(E_{11}^{2} E_{33}\right)
$$

Here $E_{11}, E_{33}, \nu_{12}, \nu_{31}$ and $G_{31}$ are the five independent elastic constants of the transversely isotropic material. Furthermore, because of the symmetry required by the existence of elastic potential:

$$
\nu_{13}=\nu_{31} E_{11} / E_{33}
$$

The macro-scale material coordinate system used to define these elastic constants is shown in Fig.2, in which $\left(x_{1}, x_{2}\right)$ is the plane of isotropy, coinciding with the bedding planes of shale.

The foregoing equations suggest that that the spherocylindrical microplane model should be able to represent the most general form of transverse orthotropy (however, in general, for materials with stronger anisotropy than shales, there is caveat, due the requirement of positiveness of microplane elastic constants; see Appendix II). The previous versions of orthotropic generalization of microplane model, particularly the weighting of microplanes (suggested in (Bažant and Oh, 1985; Bažant and Prat, 1988)) and the ellipsoidal stiffness variation depending on $\theta$ (Brocca et al., 2001), do not suffice for complete representation of orthotropy.

An exception is the spectral stiffness microplane model (Cusatis et al., 2008; Salviato et al., 2016), which does, and has been successfully applied to textile fiber-polymer composites. Nevertheless, the spectral version is conceptually more complicated and less intuitive for the modeling based on constituent properties.

In a finite loading step with microplane strain increments $\Delta \epsilon_{V}, \Delta \epsilon_{D}^{s}, \Delta \epsilon_{L}^{s}, \Delta \epsilon_{M}^{s}, \Delta \epsilon_{N}^{c}, \Delta \epsilon_{L}^{s}$ and $\Delta \epsilon_{M}^{c}$, the elastic stresses on the microplanes at the end of the loading step are obtained as

$$
\begin{aligned}
& \sigma_{V}=\sigma_{V}^{(0)}+E_{V} \Delta \epsilon_{V} \\
& \sigma_{D}^{s}=\sigma_{D}^{s(0)}+E_{D}^{s} \Delta \epsilon_{D}^{s} \\
& \sigma_{L}^{s}=\sigma_{L}^{s(0)}+E_{L}^{s} \Delta \epsilon_{L}^{s} \\
& \sigma_{M}^{s}=\sigma_{M}^{s(0)}+E_{L}^{s} \Delta \epsilon_{M}^{s} \\
& \sigma_{N}^{c}=\sigma_{N}^{c(0)}+E_{N}^{c} \Delta \epsilon_{N}^{c} \\
& \sigma_{L}^{c}=\sigma_{L}^{c(0)}+E_{L}^{c} \Delta \epsilon_{L}^{c} \\
& \sigma_{M}^{c}=\sigma_{M}^{c(0)}+E_{M}^{c} \Delta \epsilon_{M}^{c}
\end{aligned}
$$

where superscript (0) labels the initial stresses, as calculated in the previous loading step, while the elastic stresses at the end of the current loading step are labeled by no subscript. The resultant shear stresses on the spherical and cylindrical microplanes are calculated as

$$
\begin{aligned}
\sigma_{T}^{s} & =\sqrt{\left(\sigma_{L}^{s}\right)^{2}+\left(\sigma_{M}^{s}\right)^{2}} \\
\sigma_{T}^{c} & =\sqrt{\left(\sigma_{L}^{c}\right)^{2}+\left(\sigma_{M}^{c}\right)^{2}}
\end{aligned}
$$

\subsection{Remark on generalizations to full orthotropy}

If the spherocylindrical microplane model were applied to transversely orthotropic materials in which the normal stiffness and strength in the isotropy plane (i.e., in the directions normal to the cylinder axis) is lower than it is in the axis direction, the cylindrical phase stiffness would be obtained as negative. This is, of course, inadmissible. Although the case of a weaker isotropy plane does not occur for shale or other layered rocks, but materials with such a property exist, e.g., wood and many composites.

A general orthotropy, which is characterized by up to 9 independent elastic constants (and strength limits), can be achieved by two ways: 1) By weighting of the spherical microplanes 
(and strength limits) as a function of microplane dip angle (see Appendix III), and 2), in greater generality, by introducing two mutually orthogonal microplane models that are parallel to the polar direction of the spherical model; see Fig.4. For the detailed derivation of the microplane elastic constants of this configuration, see Appendix IV.

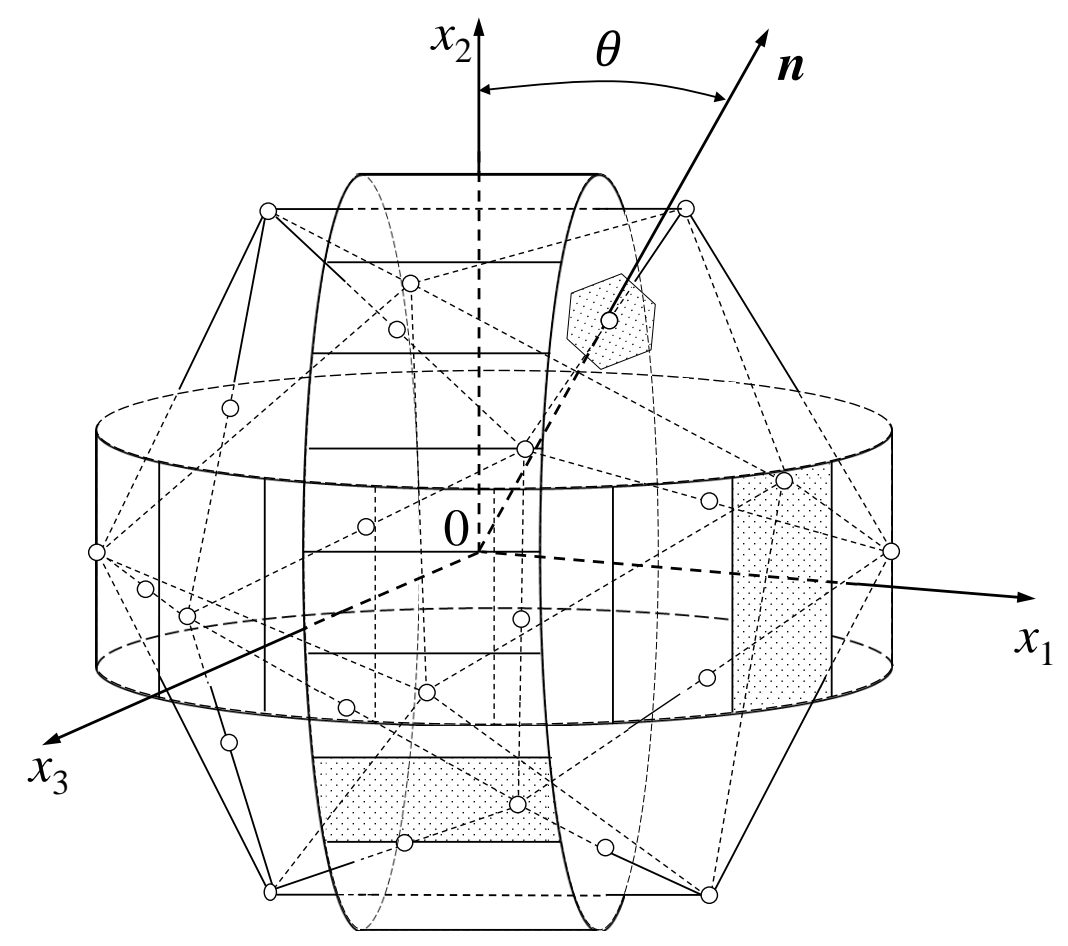

Figure 4: Microplane configuration to represent full orthotropy (note that the directions of the coordinate axes are different from the Fig.2)

\subsection{Inelastic behavior on the microplane}

Similar to the general microplane model, the inelastic behavior is characterized by the socalled stress-strain boundaries (Bažant et al., 1996), which can be regarded as strain-dependent strength limits. Within the boundaries, the response is considered to be elastic. If the boundary is exceeded in a finite time step or loading step, the stress is dropped vertically (at constant strain) to the boundary, as illustrated in Fig.5 (this is actually a special case of the classical radial return algorithm). Despite the abrupt slope change when the microplane stress reaches the boundary, the macro-scale response is quite smooth because different microplanes reach the boundary (or enter the unloading regime) at different times. The advantage of the stress-strain boundary concept is that several independent boundaries for different stress components on the same microplane can be defined (this is a major advantage over the tensorial constitutive models, in which all stress components must either load or unload simultaneously). The strain dependence of the boundaries is discussed next.

\subsubsection{Tensile normal stress boundary of the spherical phase}

The microplane normal stress boundary of the spherical phase, which limits the positive (tensile) stress $\sigma_{N}^{s}$, is imposed to simulate the tensile fracture and cracking damage. Based on the Brazilian split-cylinder test data, shown in Fig.9(a), the tensile strength is changing 


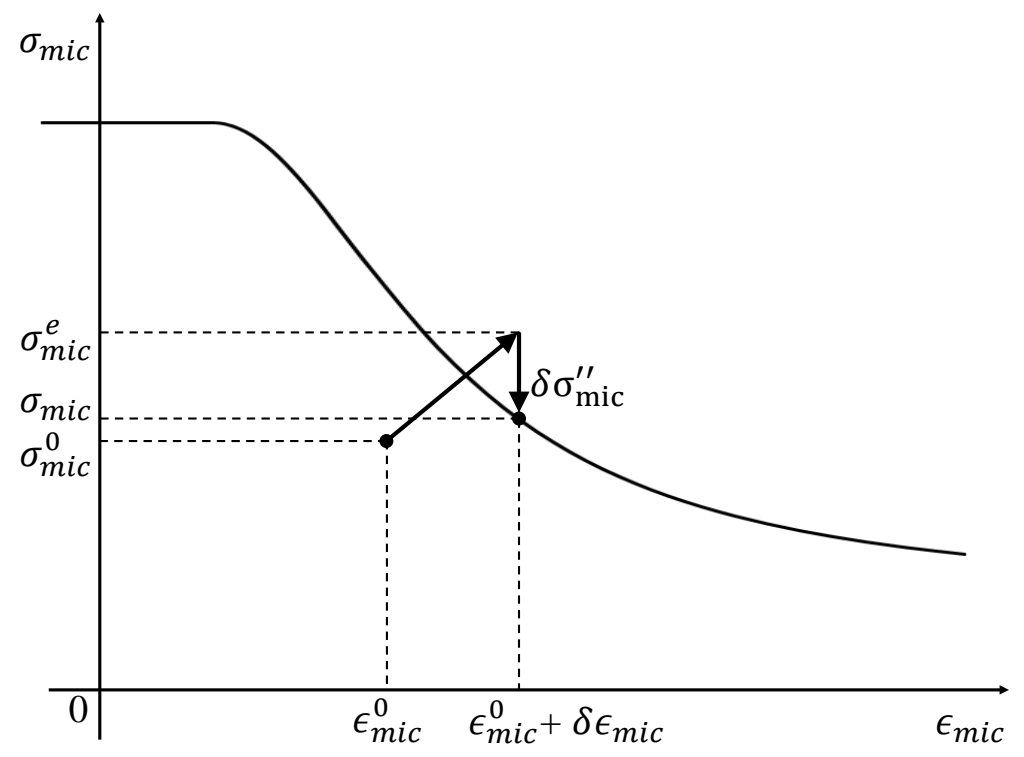

Figure 5: Schematic of vertical return to stress-strain boundary at constant strain when the boundary is exceeded by elastic stress in a finite load step.

non-monotonically as the bedding layer orientation varies. This phenomenon is described by the function (Fig.6(a))

$$
f(\theta)=1+a_{1} \sin ^{4} \theta+a_{2} \cos ^{4} \theta
$$

adjusting the boundary magnitude based on the microplane dip angle $\theta$, which is the angle between the microplane normal and axis $x_{3}$ normal to the bedding layers (Fig.2(a)). This boundary limits the positive normal stress $\sigma_{N}^{s}$ and is expressed as:

$$
\sigma_{N}^{s b+}=f(\theta) k_{t} f_{N}^{s(0)} \exp \left(-\frac{\left\langle\epsilon_{N}^{s}-\epsilon_{N}^{s(0)}\right\rangle}{k_{1} c_{3}+\left\langle-c_{4}\left(\sigma_{V} / E_{V}\right)\right\rangle}\right)
$$

Here subscript $b+$ refers to the tensile stress at the boundary; $f_{N}^{s(0)}$ is the microplane normal strength of the spherical phase, $f_{N}^{s(0)}=E_{V} k_{1} c_{1} ; \epsilon_{N}^{s(0)}$ is the microplane normal elastic strain at which the damage begins to increase, $\epsilon_{N}^{s(0)}=k_{1} c_{1} c_{2} ; k_{1}, c_{1}, c_{2}, c_{3}$ and $c_{4}$ are empirical material constants. The Macaulay brackets, defined as $\langle x\rangle=\max (x, 0)$, are used here and in what follows to define the horizontal segments of the boundaries, which, in effect, represent yield limits. See the boundary curve in Fig.7(a).

Prefactor $k_{t}$, whose default value is 1 , scales the tensile strength without significantly affecting the behavior in compression. This parameter is needed when the crack band model is used to change the element size without changing the fracture energy of propagating cracks (Bažant and Oh, 1983).

\subsubsection{Tensile and compressive deviatoric stress boundary of the spherical phase}

The tensile deviatoric stress boundary controls the lateral strain with volume expansion in weakly confined or unconfined compression. The compressive deviatoric stress boundary controls the axial crushing strain of the spherical phase in compression when the lateral confinement is too weak. As shown in Fig.9(b), the compressive strength of shale is a function of the bedding layers orientation angle. This variation tendency may be simply described by 

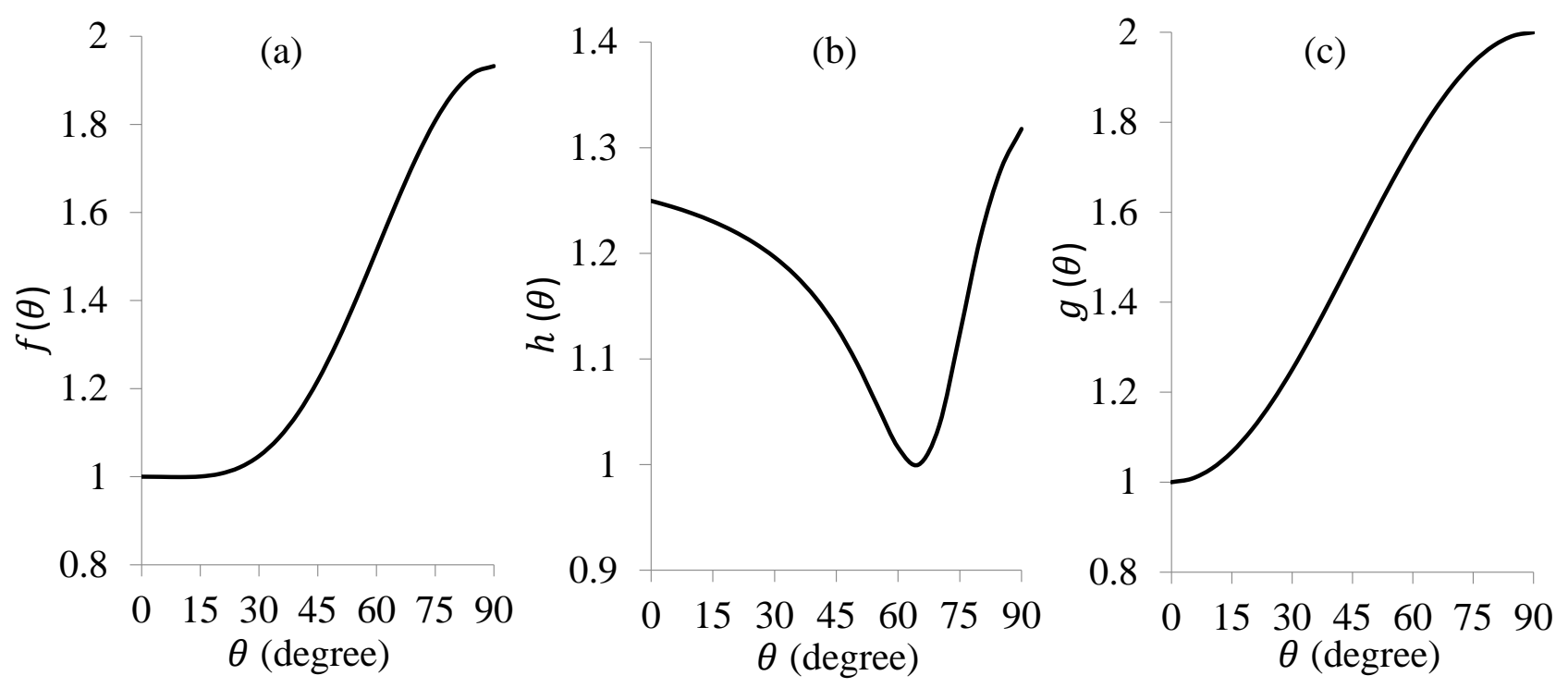

Figure 6: Schematic of the functions that are dependent on microplane dip angle $\theta$, (a) $f(\theta)$; (b) $h(\theta)$; (c) $g(\theta)$.

function ( Fig.6(b)),

$$
h(\theta)=a_{3} \cos \left(a_{4} \theta\right)+a_{5} \cos \left(a_{6} \theta\right)
$$

In compressive deviatoric boundary, function $h(\theta)$ is used to makes compressive deviatoric boundaries dependent on the microplane dip angle $\theta$. It is convenient that the same function $f(\theta)$ which is adopted in Eq.(35) may also be used for the tensile deviatoric boundary. Both boundaries have similar mathematical forms and shapes (Fig.7(b,c)):

$$
\begin{aligned}
\text { For } \sigma_{D}^{s}>0: & \sigma_{D}^{s b+}=\frac{k_{t} f(\theta) f_{D}^{0+}}{1+\left(\left\langle\epsilon_{D}^{s}-\epsilon_{D}^{s(0)+}\right\rangle / k_{1} c_{7} c_{15}\right)^{2}} \\
\text { For } \sigma_{D}^{s}<0: & \sigma_{D}^{s b-}=-\frac{h(\theta) f_{D}^{0-}}{1+\left(\left\langle-\epsilon_{D}^{s}-\epsilon_{D}^{s(0)-}\right\rangle / k_{1} c_{7}\right)^{2}}
\end{aligned}
$$

where $f_{D}^{0+}$ is the microplane tensile deviatoric strength, $f_{D}^{0+}=E_{D} k_{1} c_{5} ; \epsilon_{D}^{s(0)+}$ is the microplane tensile deviatoric strain limit; $\epsilon_{D}^{s(0)+}=k_{1} c_{5} c_{6} ; f_{D}^{0-}$ is the microplane compressive deviatoric strength, $f_{D}^{0-}=E_{D} k_{1} c_{8} ; \epsilon_{D}^{s(0)-}$ is the microplane compressive deviatoric strain limit, $\epsilon_{D}^{s(0)-}=$ $k_{1} c_{8} c_{9}$. Parameters $c_{5}, c_{6}, c_{7}, c_{8}, c_{9}$ and $c_{15}$ are empirical material constants.

\subsubsection{Shear stress boundary of the spherical phase}

On the microplane level, the shear strains $\epsilon_{L}^{s}$ and $\epsilon_{M}^{s}$ are the in-plane (or tangential) components of the vector of the projection of the macro-continuum strain tensor $\epsilon_{i j}$ onto the microplane. The dependence of shear stress-strain relation on the normal stress represents the friction, which is important to simulate the failure of material subjected to medium and high confinement. In general, the shear strength increases with the confinement, i.e, negative normal stress.

Due to insufficient data on direct shear tests of shale, the dependence of shear strength or the angular deviation of the slip plane from the bedding layers is not completely clear. Nevertheless, the bedding layers are known to be the weak layers, and so the shear strength 

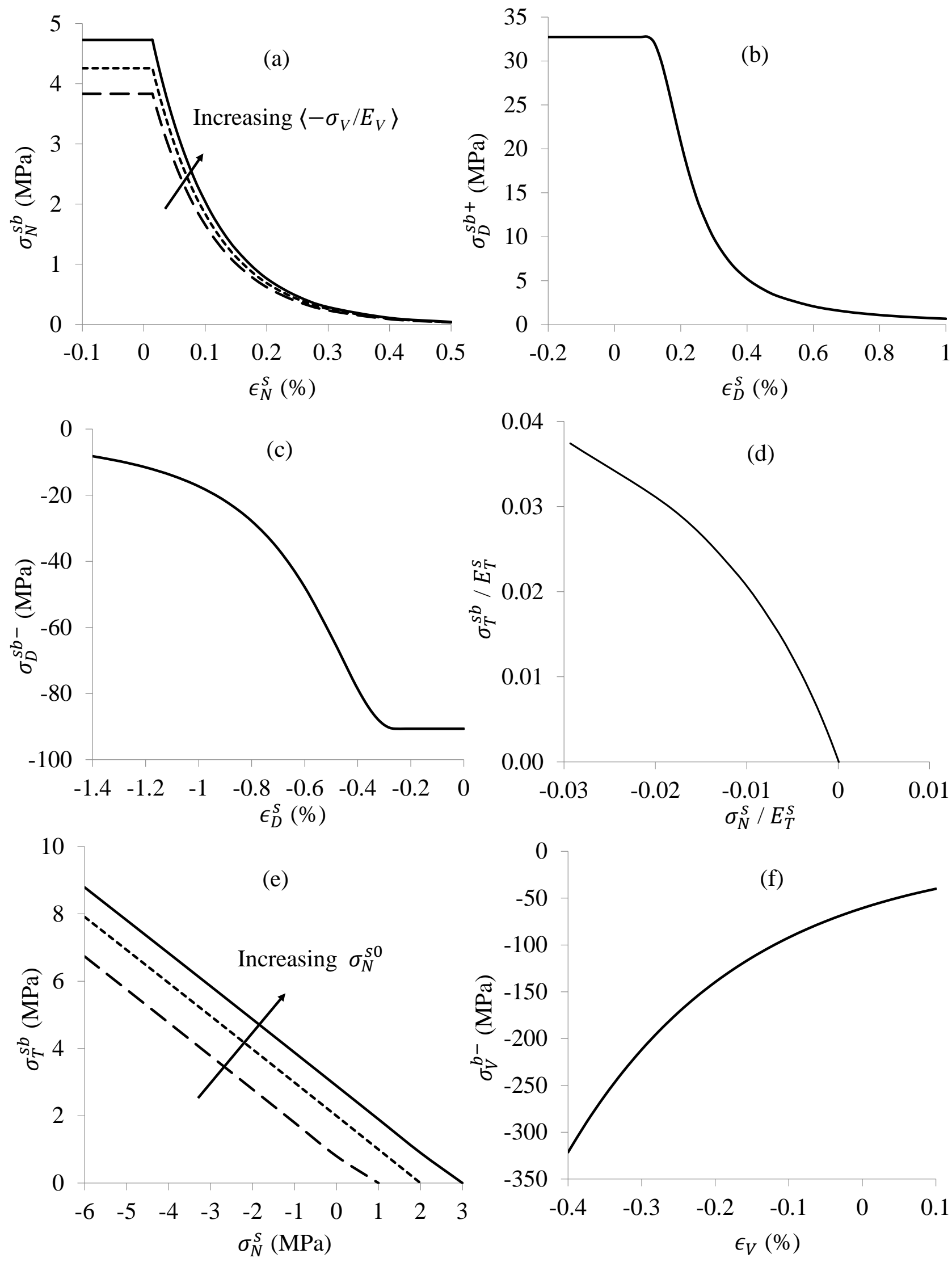

Figure 7: Diagrams of the stress-strain boundaries of the spherical phase: (a) tensile normal stress-strain boundary $\sigma_{N}^{s b+}$; (b) tensile deviatoric stress-strain boundary $\sigma_{D}^{s b+} ;$ (c) compressive deviatoric stress-strain boundary $\sigma_{D}^{s b-} ;(\mathrm{d})$ shear stress-strain boundary $\sigma_{T}^{s b}$ for broader range; (e)shear stress-strain boundary $\sigma_{T}^{s b}$ in small range; (f) compressive volumetric stress boundary $\sigma_{V}^{b-}$. 
along the bedding layers should be smallest. The dependence of the shear boundary on the microplane dip angle $\theta$ is characterized by function (see Fig. 6(c))

$$
g(\theta)=1+a_{7} \sin ^{2} \theta
$$

The shear boundary is expressed as:

$$
\begin{aligned}
\sigma_{T}^{s b} & =\frac{g(\theta) E_{T} k_{1} k_{2} c_{10}\left\langle-\sigma_{N}^{s}+\sigma_{N}^{s(0)}\right\rangle}{E_{T} k_{1} k_{2}+c_{10}\left\langle-\sigma_{N}^{s}+\sigma_{N}^{s(0)}\right\rangle} \\
\sigma_{N}^{s(0)} & =\frac{k_{t} E_{T} k_{1} c_{11}}{1+c_{12}\left\langle\epsilon_{V}\right\rangle / k_{1}}
\end{aligned}
$$

here $k_{2}, c_{10}, c_{11}$ and $c_{12}$ are empirical material constants. Eq.(40) is essentially a nonlinear Coulomb friction formula $\left((\right.$ Fig. $7(\mathrm{~d}, \mathrm{e}))$ and $\sigma_{N}^{s(0)}$ can be seen as the critical normal stress limit of the spherical phase, at which the cohesion of the spherical phase vanishes completely. As the compressive stress magnitude increases, the boundary tends to approach a horizontal asymptote, as illustrated in Fig.7(d). This is needed to simulate the material deformation when the lateral confinement is high.

\subsubsection{Compressive volumetric stress boundary of the spherical phase}

Like concrete and other quasibrittle materials, the shale subjected to pure hydrostatic compression exhibits no softening. Rather, it undergoes progressive hardening caused by closures of microcracks and voids. Similar to M4 (Bažant et al., 2000b), this feature is reflected in the following volumetric stress boundary (Fig.7(f)):

$$
\sigma_{V}^{b-}=-E_{V} k_{1} k_{3} \exp \left(-\frac{\epsilon_{V}}{k_{1} k_{4}}\right)
$$

where $k_{3}$ and $k_{4}$ are empirical material constants.

In M4 (Bažant et al., 2000b), a tensile volumetric stress boundary is also used to prevent the microplane volumetric stress from becoming too large. However, numerous experimental results indicate that this boundary has no effect on limiting the tensile volumetric stress, because the tensile stress on a generic microplane is typically limited by the tensile normal stress boundary of the spherical phase (Eq.(35)). For this reason, no tensile volumetric stress boundary of the spherical phase is introduced here.

\subsubsection{Tensile and compressive normal stress boundary of the cylindrical phase}

The tensile normal stress boundary of the cylindrical phase controls the tensile cracking in this phase. For simplicity, the mathematical form and shape of this boundary are chosen similar to those of the spherical phase. It is written as

$$
\sigma_{N}^{c b+}=f_{N}^{c(0)+} \exp \left(-\frac{\left\langle\epsilon_{N}^{c}-\epsilon_{N}^{c(0)-}\right\rangle}{k_{1} c_{3}+<-c_{4}\left(\sigma_{V} / E_{V}\right)>}\right)
$$

here $f_{N}^{c(0)}$ is the microplane normal strength of the cylindrical phase, $f_{N}^{c(0)+}=E_{N}^{c} k_{1} c_{1}$; and $\epsilon_{N}^{c(0)-}$ is the microplane normal elastic strain limit, $\epsilon_{N}^{c(0)-}=s_{1} \epsilon_{N}^{s(0)-}$ where $s_{1}$ is the scale factor to control the microplane normal elastic strain limit of the cylindrical phase. The boundary in 
Eq.(43) controls the tensile cracking and limits the additional strength in excess of that provided by the spherical microplane system. This boundary is independent of the microplane orientation $\theta$, because all the microplanes in cylindrical phase are parallel to the axis $x_{3}$ (Fig.2(b)). The diagram of Eq.(43) is shown in Fig.8(a).

The compressive normal stress boundary of the cylindrical phase controls the failure mechanism in compression of this phase when the confining pressure is not too high. It should approximately simulate the strength increases with the confining pressure. It depends on the microplane normal strain of the cylindrical phase and on the volumetric strain as follows:

$$
\sigma_{N}^{c b-}=-k_{5} f_{N}^{c(0)+}\left(1+\left(\frac{\left\langle-\epsilon_{N}^{c}-\epsilon_{N}^{c 0}\right\rangle}{c_{14}\left\langle-\epsilon_{V}\right\rangle / k_{1}}\right)^{1.5}\right)
$$

where $k_{5}$ is the ratio of the microplane compressive strength and tensile strength of the cylindrical phase; $\epsilon_{N}^{c 0+}$ is the microplane normal elastic strain limit, $\epsilon_{N}^{c 0+}=k_{1} c_{13}$; and $c_{13}, c_{14}$ are material constants. Eq.(44) dependents not only on microplane normal strain $\epsilon_{N}^{c}$ but also on volumetric strain $\epsilon_{V}$, which implies direct interaction between spherical phase and cylindrical phase. The basic form of this boundary is shown in Fig.8(b).

\subsubsection{Shear stress boundary of the cylindrical phase}

The shear stress boundary of the cylindrical phase is an analogous counterpart of the spherical phase boundary. It mainly controls the failure of the cylindrical phase when the confining pressure is high. A shear stress boundary similar to that of the spherical phase is adopted here for the cylindrical phase:

$$
\begin{aligned}
\sigma_{T}^{c b} & =\frac{s_{2} E_{M}^{c} k_{1} k_{2} c_{10}\left\langle-\sigma_{N}^{s}+\sigma_{N}^{c 0}\right\rangle}{E_{M}^{c} k_{1} k_{2}+c_{10}\left\langle-\sigma_{N}^{s}+\sigma_{N}^{c 0}\right\rangle} \\
\sigma_{N}^{c 0} & =\frac{s_{3} E_{M}^{c} k_{1} c_{11}}{1+c_{12}\left\langle\epsilon_{V}\right\rangle / k_{1}}
\end{aligned}
$$

where $\sigma_{N}^{c 0}$ can be seen as the critical normal stress limit of the cylindrical phase, at which the cohesion of the cylindrical phase vanishes completely; $s_{2}$ and $s_{3}$ are two scale parameters which change the cylindrical shear stress boundary magnitude and the critical normal stress threshold. The shear stress boundary of the cylindrical phase is shown in Fig.8(c).

\subsubsection{Unloading and reloading criteria}

To model unloading, reloading and cyclic loading, it is necessary to take into account the effect of material damage on the incremental elastic stiffness. Similar to microplane models M4 (Bažant et al., 2000b), the unloading in the microplane model is defined separately for each strain component. When any one of the products

$$
\sigma_{V} \Delta \epsilon_{V}, \quad \sigma_{D}^{s} \Delta \epsilon_{D}^{s}, \quad \sigma_{T}^{s} \Delta \epsilon_{T}^{s}, \quad \sigma_{N}^{c} \Delta \epsilon_{N}^{c}, \quad \sigma_{L}^{c} \Delta \epsilon_{L}^{c}, \quad \sigma_{M}^{c} \Delta \epsilon_{M}^{c}
$$

in the loading step becomes negative, the corresponding strain component is considered to unload. So, while one stress component is unloading, another may be loading or unloading. The following empirical equations for determining the incremental unloading moduli on the microplane are adopted:

$$
E_{V}^{U}= \begin{cases}E_{V}\left(\frac{c_{16}}{c_{16}-\epsilon_{V}}+\frac{\sigma_{V}}{c_{16} c_{17} E_{V}} \epsilon_{V}\right) & \left(\epsilon_{V}<0, \sigma_{V}<0\right) \\ \min \left(\sigma_{V} / \epsilon_{V}, E_{V}\right) & \left(\epsilon_{V}>0, \sigma_{V}>0\right)\end{cases}
$$




$$
\begin{gathered}
E_{D}^{s U}= \begin{cases}\min \left(E_{D}^{s}\left(1-c_{18}\right)+c_{18} \sigma_{D}^{s} / \epsilon_{D}^{s}, E_{D}^{s}\right) & \left(\sigma_{D}^{s}<0, E_{D}^{s} \epsilon_{D}^{s}<-E_{D}^{s} k_{1} c_{8}\right) \\
\min \left(\sigma_{D}^{s} / \epsilon_{D}^{s}, E_{D}^{s}\right) & \left(\sigma_{D}^{s}>0, E_{D}^{s} \epsilon_{D}^{s}>E_{D}^{s} k_{1} c_{5}\right)\end{cases} \\
E_{T}^{s U}= \begin{cases}\min \left(E_{T}^{s}\left(1-c_{18}\right)+c_{18} \sigma_{T}^{s} / \epsilon_{T}^{s}, E_{T}^{s}\right) & \left(\sigma_{T}^{s} \epsilon_{T}^{s}>0\right) \\
E_{T}^{s} & \left(\sigma_{T}^{s} \epsilon_{T}^{s} \leq 0\right)\end{cases} \\
E_{N}^{c U}=\left\{\begin{array}{ll}
\min \left(E_{N}^{c}\left(1-c_{18}\right)+c_{18} \sigma_{N}^{c} / \epsilon_{N}^{c}, E_{N}^{c}\right) & \left(\sigma_{N}^{s}<0, E_{N}^{c} \epsilon_{N}^{s}<-k_{5} f_{N}^{c(0)+}\right) \\
\min \left(\sigma_{N}^{c} / \epsilon_{N}^{c}, E_{N}^{c}\right) & \left(\sigma_{N}^{c}>0, E_{N}^{c} \epsilon_{N}^{c}>f_{N}^{c}(0)+\right.
\end{array}\right) \\
E_{L}^{c U}=\left\{\begin{array}{ll}
\min \left(E_{L}^{c}\left(1-c_{18}\right)+c_{18} \sigma_{L}^{c} / \epsilon_{L}^{c}, E_{L}^{c}\right) & \left(\sigma_{L}^{c} \epsilon_{L}^{c}>0\right) \\
E_{L}^{c} & \left(\sigma_{L}^{c} \epsilon_{L}^{c} \leq 0\right)
\end{array}\right) \\
E_{M}^{c U}= \begin{cases}\min \left(E_{M}^{c}\left(1-c_{18}\right)+c_{18} \sigma_{M}^{c} / \epsilon_{M}^{c}, E_{M}^{c}\right) & \left(\sigma_{M}^{c} \epsilon_{M}^{c}>0\right) \\
E_{M}^{c} & \left(\sigma_{M}^{c} \epsilon_{M}^{c} \leq 0\right)\end{cases}
\end{gathered}
$$

\subsubsection{Remarks on fracture energy, material characteristic length and shale composition}

The characteristic size, $l_{0}$, of the fracture process zone (FPZ) of shale is probably of micrometer dimensions. This is suggested by no heterogeneity visible to un-aided eye, and follows from the fact that gradual postpeak softening has never been observed on normal laboratory strength tests while, on ATM-loaded micrometer scale cantilevers, it has (Fig. 8 in (Hull et al., 2017)). The fact that the visual but the smallness of $l_{0}$ does not mean that linear elastic fracture mechanics (LEFM) could be used.

The situation looks similar to the ductile fracture of metals, where a micrometer scale FPZ is surrounded by a large plastic zone. In shale, especially under the high confinement at $3 \mathrm{~km}$ depth, the FPZ is surely surrounded by a large damage zone with gradual material softening. A large damage zone in which some microplanes are already softening develops before the peak load. In laboratory uniaxial tests, it is stable only before the peak load, although under the high triaxial confinement at $3 \mathrm{~km}$ depth it can be stable to some extent even after the peak.

The mode I fracture energy, $G_{f}$, is surely orientation dependent and, like in concrete, must strongly depend on the compressive stress parallel to the forming crack plane (which is a feature reproduced automatically by the crack band model, though not by the cohesive crack model). As in concrete, such features are approximately captured by the microplane model. The fracture energy (of dimension $\mathrm{J} / \mathrm{m}^{2}$ or $\mathrm{N} / \mathrm{m}$ ) is generally proportional to the strength limit (dimension $\mathrm{N} / \mathrm{m}^{2}$ ) multiplied by the material characteristic length $l_{0}$ (dimension $\mathrm{m}$ ).

If $l_{0}$ is much smaller than the practical width, $h$, of the crack band (equal to the finite element size), $G_{f}$ is controlled by jointly by $h$ and the vertical scaling factor of the stress-strain boundaries. For shale, the $l_{0}$ value has yet to be determined but it probably is much smaller than $1 \mathrm{~mm}$, perhaps even just a few micrometers. More fracture testing of shale is needed.

\section{Explicit numerical algorithm}

A useful feature of the present microplane model is that (like M3, M4 and M7) it is fully explicit, i.e., allows explicit calculation of stress increments from specified strain increments, and that there is no need for sub-stepping and numerical integration within the load step. This helps the numerical efficiency significantly.

The kinematic transformation matrices are computed according to Eq.(3) and Eq.(4) at the outset. This is done only once since the same matrices are used in all strain transformations. The explicit numerical algorithm within each loading step may proceed as follows: 

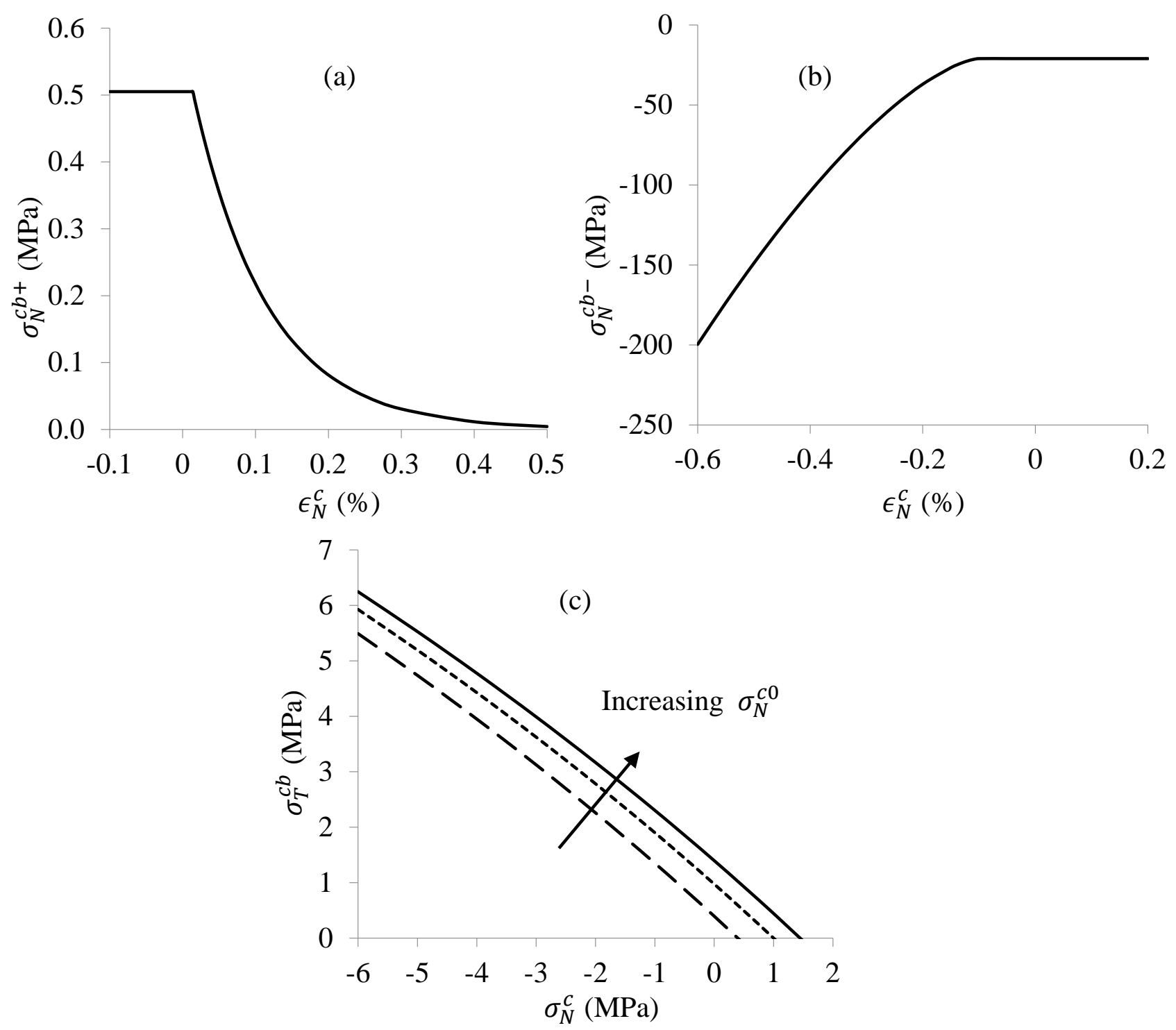

Figure 8: Diagrams of the stress-strain boundaries of the cylindrical phase: (a) tensile normal stress-strain boundary $\sigma_{N}^{c b+}$; (h) compressive normal stress-strain boundary $\sigma_{N}^{c b-}$; (i) shear stress-strain boundary $\sigma_{T}^{c b}$ over narrow range.

1. At the beginning of load step, the known quantities are the macro-continuum strain tensor $\epsilon_{i j}$, the strain increment $\Delta \epsilon_{i j}$ and the previous microplane stresses, including $\sigma_{V}, \sigma_{D}^{s}, \sigma_{L}^{s}, \sigma_{M}^{s}, \sigma_{N}^{c}$, $\sigma_{L}^{c}$ and $\sigma_{M}^{c}$, for each microplane.

2. Calculate the microplane strain increments for each microplane by using Eq.(1) and Eq.(2).

3. Check the unloading criteria to determine the microplane tangent moduli based on Eqs.(48-53).

4. Compute the microplane volumetric stress $\sigma_{V}$ (Eq.(25)) and the compressive volumetric boundary $\sigma_{V}^{b-}$ (Eq.(42)); then $\sigma_{V}^{*}=\min \left(\sigma_{V}^{e}, \sigma_{V}^{b-}\right)$.

5. Compute the microplane deviatoric stress $\sigma_{D}^{s}$ (Eq.(26)) and the tensile as well as compressive deviatoric boundaries, $\sigma_{D}^{s b}, \sigma_{D}^{s b+}$ (Eqs.(37-38)); then $\sigma_{D}^{s}=\min \left(\max \left(\sigma_{D}^{s}, \sigma_{D}^{s b}\right), \sigma_{D}^{s b+}\right)$.

6. Compute the microplane normal stress $\sigma_{N}^{s}=\sigma_{D}^{s}+\sigma_{V}$ and the tensile normal stress boundary $\sigma_{N}^{s b+}$ (Eq.(35)) of the spherical phase; then $\sigma_{N}^{s}=\min \left(\sigma_{N}^{s}, \sigma_{N}^{s b+}\right)$.

7. Recalculate the volumetric stress as the mean of the microplane normal stress $\sigma_{N}^{s}$ over 
the surface of the spherical phase, $\bar{\sigma}_{V}=\frac{1}{2 \pi} \int_{\Omega} \sigma_{N}^{s} d_{\Omega}$; then $\sigma_{V}=\min \left(\bar{\sigma}_{V}, \sigma_{V}^{*}\right)$.

8. Recalculate the microplane deviatoric stress of the spherical phase by using $\sigma_{D}^{s}=\sigma_{N}^{s}-\sigma_{V}$.

9. Compute the microplane shear stresses $\sigma_{L}^{s}$ (Eq.(27)), $\sigma_{M}^{s}$ (Eq.(28)) of the spherical phase, and the resultant of those two shear stresses $\sigma_{T}^{s}$ (Eq.(32)). Also, calculate the shear stress boundary $\sigma_{T}^{s b}$ (Eq.(40)). Then set $\sigma_{T}^{s *}=\min \left(\sigma_{T}^{s}, \sigma_{T}^{s b}\right)$. Then obtain $\sigma_{L}^{s}=\sigma_{T}^{s *} \sigma_{L}^{s} / \sigma_{T}^{s}$ and $\sigma_{M}^{s}=\sigma_{T}^{s *} \sigma_{M}^{s} / \sigma_{T}^{s}$.

10. Calculate the microplane normal stress $\sigma_{N}^{c}$ (Eq.(29)) and tensile as well as compressive normal boundary of the cylindrical phase, $\sigma_{N}^{c b+}$ (Eq.(43)), $\sigma_{N}^{c b-}$ (Eq.(44)). Then get $\sigma_{N}^{c}=$ $\min \left(\max \left(\sigma_{N}^{c}, \sigma_{N}^{c b-}\right), \sigma_{N}^{c b+}\right)$.

11. Compute the microplane shear stresses $\sigma_{L}^{c}$ (Eq.(30)), $\sigma_{M}^{c}$ (Eq.(31)) of the cylindrical phase, and the resultant of those two shear stresses $\sigma_{T}^{c}$ (Eq.(33)). Also calculate the shear stress boundary $\sigma_{T}^{c b}$ (Eq.(45)). Then set $\sigma_{T}^{c *}=\min \left(\sigma_{T}^{c}, \sigma_{T}^{c b}\right)$. Then obtain $\sigma_{L}^{c}=\sigma_{T}^{c *} \sigma_{L}^{c} / \sigma_{T}^{c}$ and $\sigma_{M}^{c}=\sigma_{T}^{c *} \sigma_{M}^{c} / \sigma_{T}^{c}$.

12. Update $\sigma_{V}, \sigma_{N}^{s}, \sigma_{D}^{s}, \sigma_{L}^{s}$ and $\sigma_{M}^{s}$ for each microplane of the spherical phase. Update $\sigma_{N}^{c}, \sigma_{L}^{c}$ and $\sigma_{M}^{c}$ for each microplane of the cylindrical phase.

13. Calculate the macro-continuum stress tensor $\sigma_{i j}$ according to Eq.(7).

Based on the algorithm introduced above, this new model is written as a subroutine and is implemented into ABAQUS via users' subroutine interface VUMAT, to calibrate the parameters and predict the shale deformation.

\section{Verification and calibration by experimental data}

Longmaxi shale, from outcrops in Pengshui county, Chongqing. China, was tested at Sichuan University using MTS-815 electro-hydraulic servo-controlled rock mechanics testing system. The specimens were cylinders of diameter $50 \mathrm{~mm}$ and length $100 \mathrm{~mm}$. To study anisotropy, the program included seven different bedding layer inclinations with respect to cylinder axis, $\beta=0^{\circ}, 15^{\circ}, 30^{\circ}, 45^{\circ}, 60^{\circ}, 75^{\circ}, 90^{\circ}$, and standard triaxial compression tests with four levels of lateral confinement: $\sigma_{3}=10 \mathrm{MPa}, 30 \mathrm{MPa}, 45 \mathrm{MPa}, 60 \mathrm{MPa}$. All the triaxial compression tests were repeated once. The program further included uniaxial compression tests $\left(\beta=0^{\circ}\right.$, $90^{\circ}$ ) and Brazilian split-cylinder tensile strength tests with $\beta=0^{\circ}, 15^{\circ}, 30^{\circ}, 45^{\circ}, 60^{\circ}, 75^{\circ}, 90^{\circ}$, which are repeated twice to reduce experimental error.

The tests revealed one important feature - the strength variation from normal to parallel direction is non-monotonic, with a minimum compressive strength occurs as the bedding layer orientation angle is $60^{\circ}$. The variation of Brazilian tensile strength and compressive strength with bedding layer orientation is shown in Fig. 9. The scatter of compressive strength values is very small. The maximum deviation from the mean was only about $5 \%$, which is very small relative to the minimum compressive strength. The coefficients of variation of Brazilian Split-cylinder tests for $\beta=0^{\circ}, 15^{\circ}, 30^{\circ}, 45^{\circ}, 60^{\circ}, 75^{\circ}$ and $90^{\circ}$ are $16.7 \%, 12.3 \%, 13.5 \%, 11.3 \%$, $9.3 \%, 8.8 \%, 8.6 \%$, respectively.

To validate the model, only a part of the experimental data set is used here to identify, by optimum fitting, the spherocylindrical model parameters. The remaining art is then used to compare the prediction results.

\subsection{Identification of model parameters by fitting a part of Longmaxi data set}

Although there are many parameters in the spherocylindrical microplane model, calibration is greatly facilitated by exploiting similarities with the previous microplane models for concrete and isotropic rock, particularly (Bažant and Zi, 2003; Caner and Bažant, 2000). First the five independent elastic constants, $E_{11}, E_{33}, \nu_{12}, \nu_{31}$ and $G_{31}$, are easily determined from the uniaxial 

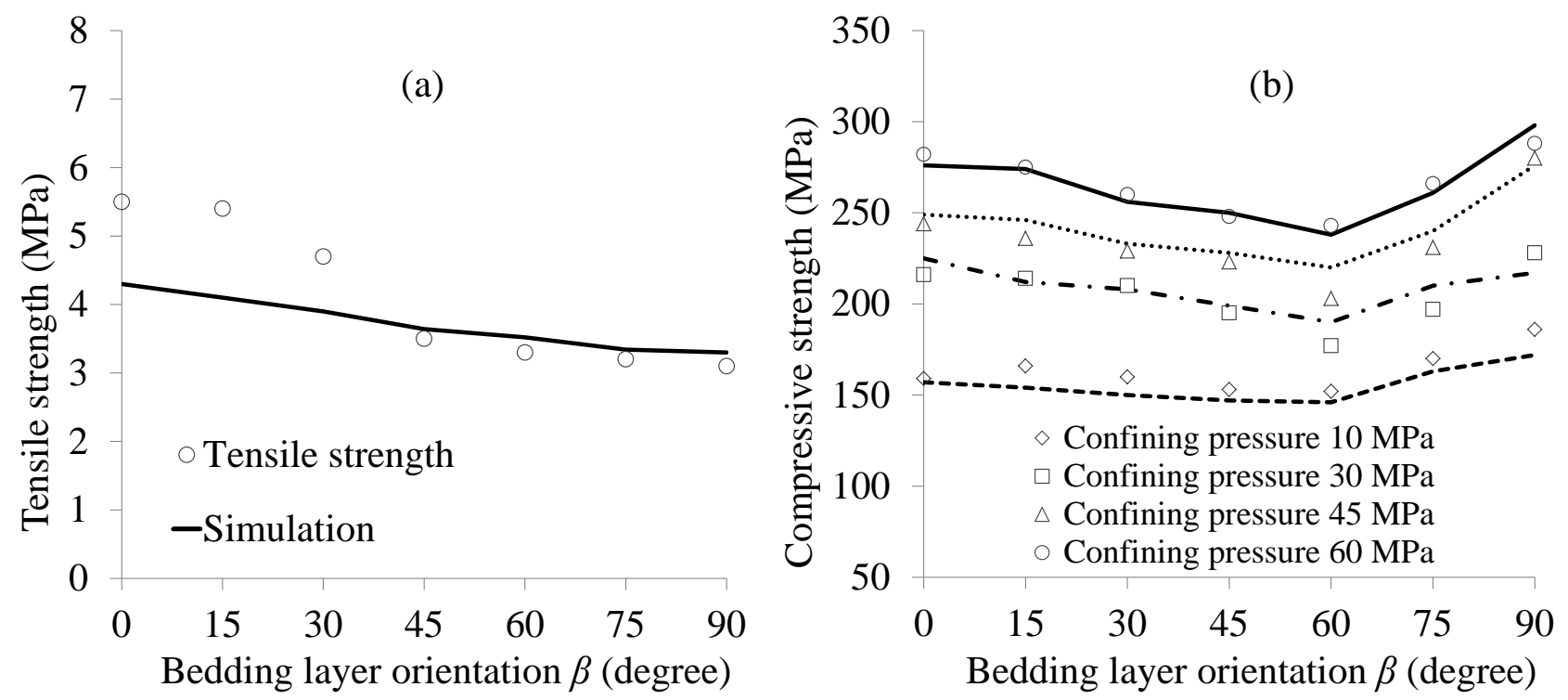

Figure 9: (a) Brazilian split-cylinder tensile strength; (b) triaxial compressive strength (the data points are the averages of test data, and the lines represent the simulation results).

compression tests of Longmaxi shale with different bedding layer orientations. Then the elastic constants for the microplanes are calculated from Eqs.(12-17). The coefficients in functions $f(\theta)$, $h(\theta)$ and $g(\theta)$ are then obtained by fitting the variation of tensile and compressive strengths as a function of the bedding layer orientation.

From the hydrostatic compression curve, one can then identify the magnitudes of products $k_{1} k_{3}, k_{1} k_{4}$ as well as parameters $c_{16}$ and $c_{17} ; k_{3}$ controls the strength threshold at which the microcracks or micropores pore begin to collapse; $k_{4}$ controls the subsequent hardening rate (or steepness); $c_{16}$ and $c_{17}$ control the volumetric unloading modulus.

The way to determine the value of $k_{1}$ is by fitting of the stress-strain curve for uniaxial tension. Because no direct tensile test has been carried out for the Longmaxi shale, $k_{1}$ is simply determined from the tensile Brazilian split-cylinder strength $\left(\beta=0^{\circ}\right)$. Then, in combination with the Brazilian test at $\beta=90^{\circ}, c_{1}, c_{2}$ and $s_{1}$ can be identified; $c_{3}$ and $c_{4}$ control the postpeak softening in direct tension and, in absence of the direct tensile tests, these two parameters have to be estimated from test data for similar geomaterials.

Fitting the uniaxial compression tests at $\beta=0^{\circ}$, one can get values of $c_{5}, c_{6}, c_{7}, c_{8}, c_{9}, c_{15}$ and $c_{18}$, among which $c_{5}$ and $c_{6}$ control the microplane strength; $c_{6}$ and $c_{9}$ control the damage initiation; and $c_{7}$ and $c_{15}$ can control the post peak softening curve in compression; Then the values of $s_{1}, c_{13}, c_{14}, k_{5}$ are calibrated according to the uniaxial compression $\left(\beta=90^{\circ}\right)$.

The way to calibrate the $c_{10}, c_{11}, c_{12}, s_{2}, s_{3}$ and $k_{2}$ is to match the direct shear test data for various bedding layer orientations. For lack of relevant test data, the triaxial compression test at $\beta=0^{\circ}$ and confining pressure $60 \mathrm{MPa}$ is used instead, to determine the values of $c_{10}, c_{11}, c_{12}$ and $k_{2}$. Then the two scale factors $s_{2}$ and $s_{3}$ are obtained from the triaxial compression test at $\beta=90^{\circ}$ and confining pressure $60 \mathrm{MPa}$.

All the parameters $c_{1}, c_{2}, \ldots, c_{18}$ are all dimensionless, which does not preclude their approximate applicability to other shale, especially when the mineral content is similar (although calibration tests are always desirable). The strength and other basic characteristics of the shale are controlled by five adjustable parameters like general microplane model: $k_{1}$ governs radial scaling of the stress-strain curves; $k_{2}$ controls frictional strength: $k_{3}$ ultimates the magnitude of hydrostatic boundary; $k_{4}$ controls the slope of the hydrostatic boundary and $k_{5}$ governs 
compressive strength of cylindrical phase.

For Longmaxi shale, the optimized easily scalable parameters are $k_{1}=2.55 D^{-4}, k_{2}=260$, $k_{3}=7.1, k_{4}=28.2$ and $k_{5}=6$, respectively. The optimized hard-to-adjust parameters, $c_{1}, c_{2}, \ldots, c_{18}$, are $0.2,2.5,2.5,70,8,20,1.3,12,1.2,3.0,3.2,5,3.1,9.5,0.35,0.02,0.01,0.4$, respectively. The measured elastic constants based on uniaxial compression tests are $E_{11}=28.2$ $\mathrm{GPa}, E_{33}=24.2 \mathrm{GPa}, \nu_{12}=0.11, \nu_{31}=0.138, G_{31}=13.5 \mathrm{GPa}$ and $E_{11} / E_{33}=1.17$.

All the calibration curves and their comparisons with experimental data points are shown in Fig. 10.

\subsection{Comparison of the spherocylindrical model predictions with the remaining part of Longmaxi data set}

To evaluate the performance of the spherocylindrical model, the series of triaxial tests at different initial confinements and for various bedding layer orientations is subsequently simulated. The shale sample numbering may be explained by an example: "30-60" means that the bedding layer inclination with respect to cylinder axis is $\beta=30^{\circ}$ and the confining pressure is $60 \mathrm{MPa}$. The comparisons between the prediction results and the experimental data points are provided in Fig. 9 and Fig. 12.

Generally, the proposed model can capture the strength and main mechanical properties of anisotropic shale quite well. As expected, the ultimate strength usually increases and the inelastic deformation becomes more pronounced as the confining pressure is raised.

To calculate the splitting tensile strength, the standard formula for isotropic materials has been used. Because the Brazilian split-cylinder test generally gives only a crude estimate of the direct tensile strength and gives rather scattered results, especially for shale, the simple standard formula for isotropic elastic cylinders has been adopted for a simple estimate of the tensile strength, in preference over a complicated calculation of quasibrittle splitting fracture evolution taking into account the orthotropy, a curved shape of splitting crack for inclined bedding layer orientations and development of damage before the peak load.

For angles $<30^{\circ}$, the standard formula for the split-cylinder tensile strength is seen in the figure to give bigger errors. Aside from the error of the standard splitting strength formula (Vervoort et al., 2014), the main cause of the deviations from test data seen in this range is the aforementioned complex failure mechanism, with a curved crack. Finite element analysis of the orthotropic Brazilian test would be needed to clarify it in detail, but this must be relegated to future study.

Much of the smaller scatter represents not only the inevitable experimental error and shale randomness but also by complex effects of the bedding layers on the mechanical behavior. For instance, the specimen 75-30 has a smaller peak strain and strength than the specimen 75-10 with a much weaker confinement. This might not be just by chance.

Another source of error is that while some microplanes exhibit gradual softening before the peak load, a stable postpeak softening of the test specimens of shale has never been observed in experiments and probably does not exist at normal laboratory scale, as already discussed in connection with $l_{0}$ in a previous section. The test specimens were obviously not small enough to avoid localization of damage, which destabilizes the test (Bažant, 1976). To avoid it, the specimens would have to be of sub-millimeter size. However, there is another way to identify unambiguously the postpeak from experiments - conduct size effect tests coupled with scale fracture tests of notched specimens (Hoover and Bažant, 2014).

The microplane postpeak softening is important not only for the overall material softening, but also for prepeak hardening. Many individual microplanes undergo postpeak softening already before the peak load of the specimen is reached. Thus the microplane postpeak controls 
the decrease of the rising prepeak slope. Consequently, fitting the material prepeak response helps identifying the softening parameters at least partly.

To demonstrate the necessity of making the stress-strain boundaries dependent, through functions $f(\theta), h(\theta)$ and $g(\theta)$, on the microplane dip angle $\theta$, one simulation without using these three functions is conducted and then compared with the simulation results obtained with the normal boundaries, as shown in Fig. 11. As can be seen, functions $f(\theta), h(\theta)$ and $g(\theta)$ have significant influence not only on the peak strain and strength, but also on the inelastic deformation, and especially on the softening curve. It is necessary to introduce realistic forms of functions $f(\theta), h(\theta)$ and $g(\theta)$ into the stress-strain boundaries, so as to simulate the real mechanical behavior of shale.

\subsection{Adjustment of material parameters to characterize Tournemire shale}

To characterize other shales, the spherocylindrical model parameters need to be adjusted. However, similar to concretes, it appears that only a few parameters need adjustment. To provide a further validation of the predictive ability of the spherocylindrical model, the test data for Tournemire shale (Masri et al., 2014) may be used.

All the parameters $c_{1}, c_{2} \ldots, c_{18}$ in the present model are all dimensionless. Thus they are likely to work for other shales and are kept unchanged. But the dimensionless functions $f(\theta)$, $h(\theta)$ and $g(\theta)$ need to be changed according to strength variations of Tournemire shale, which are different and are functions of the loading direction relative to the bedding layers. The elastic moduli of Tournemire shale under uniaxial compression are $E_{11}=19.3 \mathrm{GPa}, E_{33}=10.0 \mathrm{GPa}$, $\nu_{12}=0.15, \nu_{31}=0.24, G_{31}=5.5 \mathrm{GPa}$ and $E_{11} / E_{33}=1.93$.

The Tournemire specimens 0-20 and 90-20 (for which the previous rule of shale sample numbering is retained) are used to calibrate parameters $k_{1}, k_{2}, \ldots, k_{5}$, and $k_{1}=1.47 D^{-4}, k_{2}=$ $140, k_{3}=6.5, k_{4}=36, k_{5}=1.8$. Then the remaining Tournemire shale data are used to check the prediction; see Fig.13. As can be seen, the tests and predictions are satisfactory.

\section{Conclusions}

1. Unlike the previous anisotropic generalizations of the microplane model except the spectral model, the spherocylindrical microplane model proposed here can reproduce all the five independent elastic constants of transversely isotropic shales. The elastic moduli for the spherical and cylindrical microplanes can be calculated easily from these five constants.

2. The moduli must all be positive. This is true if and only if the elastic in- to out-of-plane moduli ratio (or degree of anisotropy) is not too high, usually less than 3.75 which appears to be true for all known shales.

3. Since oriented crack openings, frictional slips and bedding plane orientations are directly reflected on the microplanes, the inelastic behavior of the present model can be formulated more easily and intuitively than in the spectral microplane model.

4. The resistance to slip and cracking, governed on the microplanes by the stress-strain boundaries (or strain-dependent strength limits), depends strongly and non-monotonically on the dip angle $\theta$ (i.e., the angle between the microplane normal direction and the loading direction).

5. The cylindrical microplanes capture effectively the increase of stiffness in the directions along the bedding layers of shale. The spherical model alone appears incapable of fitting all the experimental behavior considered here, even if the microplane weights and the constitutive laws are made to depend on angle $\theta$. 
6. Thanks to the kinematic constraint of both the spherical and cylindrical microplanes and the formulation of computational algorithm, the finite element program using the present model is fully explicit. This helps computational efficiency and robustness.

7. Experiments and numerical simulations are conducted for triaxial compression tests of shale with different confining pressures as well as bedding layer orientations. The comparisons between the experimental data and numerical predictions show that the proposed model can capture the main features of the mechanical behavior of anisotropic shales.

8. Although the present model is calibrated for two kinds of shale only, it appears that by scaling a few parameters it can give good predictions for other shales, although more calibration tests may be needed.

\section{Appendix}

Appendix I: Calculation of microplane elastic moduli

For clarity, this Appendix describes the construction of the complete stiffness tensor $\bar{C}_{i j k l}$. The Voigt notation is adopted here, which allows writing the symmetric fourth-order tensor as a 6 by 6 symmetric matrix. In particular, $N_{i j}^{s} N_{k l}^{s}=\bar{N}_{I}^{s} \bar{N}_{J}^{s}, L_{i j}^{s} L_{k l}^{s}=\bar{L}_{I}^{s} \bar{L}_{J}^{s}, M_{i j}^{s} M_{k l}^{s}=\bar{M}_{I}^{s} \bar{M}_{J}^{s}$, $N_{i j}^{s} \Delta_{k l}=\bar{N}_{I}^{s} \bar{\Delta}_{J}, N_{i j}^{c} N_{k l}^{c}=\bar{N}_{I}^{c} \bar{N}_{J}^{c}, L_{i j}^{c} L_{k l}^{c}=\bar{L}_{I}^{c} \bar{L}_{J}^{c}$ and $M_{i j}^{c} M_{k l}^{c}=\bar{M}_{I}^{c} \bar{M}_{J}^{c}$, where $I, J=1,2, \ldots, 6$. Then, based on the positions of the microplanes, the following matrices are obtained:

$$
\begin{gathered}
\int_{\Omega} \bar{N}_{I}^{s} \bar{N}_{J}^{s} d \Omega=\frac{2 \pi}{15}\left(\delta_{i j} \delta_{k l}+\delta_{i k} \delta_{j l}+\delta_{i l} \delta_{j k}\right)=\frac{2 \pi}{3}\left[\begin{array}{cccccc}
0.6 & 0.2 & 0.2 & 0 & 0 & 0 \\
0.2 & 0.6 & 0.2 & 0 & 0 & 0 \\
0.2 & 0.2 & 0.6 & 0 & 0 & 0 \\
0 & 0 & 0 & 0.2 & 0 & 0 \\
0 & 0 & 0 & 0 & 0.2 & 0 \\
0 & 0 & 0 & 0 & 0 & 0.2
\end{array}\right] \\
\int_{\Omega}\left(\bar{L}_{I}^{s} \bar{L}_{J}^{s}+\bar{M}_{I}^{s} \bar{M}_{J}^{s}\right) d \Omega=\frac{\pi}{15}\left(-2 \delta_{i j} \delta_{k l}+3 \delta_{i k} \delta_{j l}+3 \delta_{i l} \delta_{j k}\right)=\frac{2 \pi}{3}\left[\begin{array}{cccccc}
0.4 & -0.2 & -0.2 & 0 & 0 & 0 \\
-0.2 & 0.4 & -0.2 & 0 & 0 & 0 \\
-0.2 & -0.2 & 0.4 & 0 & 0 & 0 \\
0 & 0 & 0 & 0.3 & 0 & 0 \\
0 & 0 & 0 & 0 & 0.3 & 0 \\
0 & 0 & 0 & 0 & 0 & 0.3
\end{array}\right]
\end{gathered}
$$

$$
\begin{gathered}
\int_{\Omega} \bar{N}_{I}^{s} \bar{\Delta}_{J} d \Omega=\frac{2 \pi}{3}\left(\delta_{i j} \delta_{k l}\right)=\frac{2 \pi}{3}\left[\begin{array}{llllll}
1 & 1 & 1 & 0 & 0 & 0 \\
1 & 1 & 1 & 0 & 0 & 0 \\
1 & 1 & 1 & 0 & 0 & 0 \\
0 & 0 & 0 & 0 & 0 & 0 \\
0 & 0 & 0 & 0 & 0 & 0 \\
0 & 0 & 0 & 0 & 0 & 0
\end{array}\right] \\
\int_{S} \bar{N}_{I}^{c} \bar{N}_{J}^{c} d S=\frac{1}{N_{m}^{c}} \sum_{\mu=1}^{N_{m}^{c}} \bar{N}_{I}^{c} \bar{N}_{J}^{c}=\pi\left[\begin{array}{cccccc}
0.375 & 0.125 & 0 & 0 & 0 & 0 \\
0.125 & 0.375 & 0 & 0 & 0 & 0 \\
0 & 0 & 0 & 0 & 0 & 0 \\
0 & 0 & 0 & 0 & 0 & 0 \\
0 & 0 & 0 & 0 & 0 & 0 \\
0 & 0 & 0 & 0 & 0 & 0.125
\end{array}\right]
\end{gathered}
$$




$$
\begin{gathered}
\int_{S} \bar{L}_{I}^{c} \bar{L}_{J}^{c} d S=\frac{1}{N_{m}^{c}} \sum_{\mu=1}^{N_{m}^{c}} \bar{L}_{I}^{c} \bar{L}_{J}^{c}=\pi\left[\begin{array}{cccccc}
0.125 & -0.125 & 0 & 0 & 0 & 0 \\
-0.125 & 0.125 & 0 & 0 & 0 & 0 \\
0 & 0 & 0 & 0 & 0 & 0 \\
0 & 0 & 0 & 0 & 0 & 0 \\
0 & 0 & 0 & 0 & 0 & 0 \\
0 & 0 & 0 & 0 & 0 & 0.125
\end{array}\right] \\
\int_{S} \bar{M}_{I}^{c} \bar{M}_{J}^{c} d S=\frac{1}{N_{m}^{c}} \sum_{\mu=1}^{N_{m}^{c}} \bar{M}_{I}^{c} \bar{M}_{J}^{c}=\pi\left[\begin{array}{cccccc}
0 & 0 & 0 & 0 & 0 & 0 \\
0 & 0 & 0 & 0 & 0 & 0 \\
0 & 0 & 0 & 0 & 0 & 0 \\
0 & 0 & 0 & 0.125 & 0 & 0 \\
0 & 0 & 0 & 0 & 0.125 & 0 \\
0 & 0 & 0 & 0 & 0 & 0
\end{array}\right]
\end{gathered}
$$

In Voigt notation, the transverse isotropic stiffness tensor $\bar{C}_{i j k l}$ can be written as a matrix $C_{I J}(I, J=1,2, \ldots 6)$

$$
C_{I J}=\left[\begin{array}{cccccc}
C_{11} & C_{12} & C_{13} & 0 & 0 & 0 \\
C_{12} & C_{11} & C_{13} & 0 & 0 & 0 \\
C_{13} & C_{13} & C_{33} & 0 & 0 & 0 \\
0 & 0 & 0 & C_{44} & 0 & 0 \\
0 & 0 & 0 & 0 & C_{44} & 0 \\
0 & 0 & 0 & 0 & 0 & C_{66}
\end{array}\right]
$$

For transversely isotropic materials, $C_{11}=C_{12}+2 C_{66}, C_{11}=C_{22}, C_{13}=C_{23}$ and $C_{44}=C_{55}$.

The two shear moduli of the spherical microplanes must be equal, i.e., $E_{L}^{s}=E_{M}^{s}\left(=E_{T}^{s}\right)$. Furthermore, it is assumed that $E_{D}^{s}=\eta E_{L}^{s}$ and $\eta=1$. Substituting Eqs.(54-59) into Eq.(11), and noting the analogy with Eq.(60), one has, one obtains:

$$
\begin{aligned}
& \alpha\left[0.6 E_{D}^{s}+\frac{1}{3}\left(E_{V}-E_{D}^{s}\right)+0.4 E_{L}^{s}\right]+(1-\alpha)\left(0.375 E_{N}^{c}+0.125 E_{L}^{c}\right)=C_{11} \\
& \alpha\left[0.2 E_{D}^{s}+\frac{1}{3}\left(E_{V}-E_{D}^{s}\right)-0.2 E_{L}^{s}\right]+(1-\alpha)\left(0.125 E_{N}^{c}-0.125 E_{L}^{c}\right)=C_{12} \\
& \alpha\left[0.2 E_{D}^{s}+\frac{1}{3}\left(E_{V}-E_{D}^{s}\right)-0.2 E_{L}^{s}\right]=C_{13} \\
& \alpha\left[0.6 E_{D}^{s}+\frac{1}{3}\left(E_{V}-E_{D}^{s}\right)+0.4 E_{L}^{s}\right]=C_{33} \\
& \alpha\left(0.2 E_{D}^{s}+0.3 E_{L}^{s}\right)+(1-\alpha) 0.125 E_{M}^{c}=C_{44}
\end{aligned}
$$

Based on Eqs.(61-65), the elastic parameters on the microplane Eq.(12-17) can be obtained.

Appendix II: Reasons for adopting the volumetric-deviatoric split

The volumetric-deviatoric split means that the microplane normal strain $\epsilon_{N}^{s}$ in the spherical microplane is considered as the sum of the volumetric strain $\epsilon_{V}$ and the deviatoric strain $\epsilon_{D}^{s}$ (Eq.(5)). One reason for the split is that the model must simultaneously capture two facts: (i) confined or weakly confined compression exhibits a maximum load and postpeak softening, while (ii) hydrostatic or perfectly confined compression does not. Both cannot be attained without the split. The second reason is that the isotropic spherical system without the split cannot exhibit Poisson's ratio greater than 0.25 (for similar reasons as posited already by Poisson himself in the mid 1800s), while the split allows modeling Poisson's ratios up to 0.5. 
For the spherocylindrical model, a similar limitation must be overcome. Without the volumetric-deviatoric split, the elastic constitutive model on the microplane is

$$
\sigma_{N}^{s}=E_{N}^{s} \epsilon_{N}^{s}, \sigma_{L}^{s}=E_{L}^{s} \epsilon_{L}^{s}, \sigma_{M}^{s}=E_{M}^{s} \epsilon_{M}^{s}, \sigma_{N}^{c}=E_{N}^{c} \epsilon_{N}^{c}, \sigma_{L}^{c}=E_{L}^{c} \epsilon_{L}^{c}, \sigma_{M}^{c}=E_{M}^{c} \epsilon_{M}^{c}
$$

where $E_{N}^{s}, E_{L}^{s}, E_{M}^{s}, E_{N}^{c}, E_{L}^{c}$ and $E_{M}^{c}$ are the microplane elastic moduli.

Substitution of Eqs.(1-2) and Eq.(66) into Eq.(6), the macroscopic transversely isotropic stiffness tensor $\bar{C}_{i j k l}$ is obtained:

$$
\begin{aligned}
\bar{C}_{i j k l}= & 6 \alpha \sum_{\mu=1}^{N_{m}^{s}} w_{\mu}^{s}\left(E_{N}^{s} N_{i j}^{s} N_{k l}^{s}+E_{L}^{s} L_{i j}^{s} L_{k l}^{s}+E_{M}^{s} M_{i j}^{s} M_{k l}^{s}\right)^{(\mu)} \\
& +(1-\alpha) \frac{1}{\pi N_{m}^{c}} \sum_{\mu=1}^{N_{m}^{c}}\left(E_{N}^{c} N_{i j}^{c} N_{k l}^{c}+E_{L}^{c} L_{i j}^{c} L_{k l}^{c}+E_{M}^{c} M_{i j}^{c} M_{k l}^{c}\right)^{(\mu)}
\end{aligned}
$$

Based on the methodology and results introduced in Appendix I, the microplane elastic constants without consideration of the volumetric-deviatoric split are computed as

$$
\begin{aligned}
& E_{N}^{s}=\left(C_{33}+2 C_{13}\right) / \alpha \\
& E_{L}^{s}=E_{M}^{s}=E_{T}^{s}=\left(C_{33}-3 C_{13}\right) / \alpha \\
& E_{N}^{c}=2\left(C_{11}-C_{33}+C_{12}-C_{13}\right) /(1-\alpha) \\
& E_{L}^{c}=2\left(C_{11}-C_{33}-3 C_{12}+3 C_{13}\right) /(1-\alpha) \\
& E_{M}^{c}=4\left(2 C_{44}-C_{33}+C_{13}\right) /(1-\alpha)
\end{aligned}
$$

It is found that Eq.(68) and Eqs.(70-72) are usually greater than 0, and so it suffices to check Eq.(69). Substituting Eqs.(20-21) into Eq.(69), one can see that if $E_{L}^{s}$ is greater than 0, the macro-scale elastic parameters must satisfy the condition:

$$
\zeta_{1}=\frac{E_{33}}{E_{11}}\left(1-\nu_{12}\right)-3 \nu_{31}>0
$$

Here the in- to out-of-plane ratio $E_{11} / E_{33}$ characterizes the the degree of anisotropy.

The macro-scale elastic constants of many shales tests (Sone, 2012) have been used to check inequality (73), as shown table 1. It is seen that this inequality is satisfied only when both the degree of anisotropy and Poisson's ratio are small. However, this is frequently not the case. Hence, try the split.

Table 1: The values of $\zeta_{1}$ and $\zeta_{2}$ for different shale tests.

\begin{tabular}{cccccccccccc}
\hline$E_{33}$ & 13.8 & 17.6 & 18.5 & 15.4 & 16.7 & 10.9 & 10.7 & 8.8 & 14.2 & 15 & 12.3 \\
$E_{11}$ & 40.4 & 34.4 & 40.5 & 31.5 & 29.2 & 29.4 & 28.4 & 33 & 28.2 & 28.9 & 29.0 \\
$\nu_{31}$ & 0.13 & 0.19 & 0.17 & 0.14 & 0.21 & 0.19 & 0.16 & 0.26 & 0.22 & 0.23 & 0.15 \\
$\nu_{12}$ & 0.34 & 0.30 & 0.27 & 0.19 & 0.24 & 0.24 & 0.24 & 0.32 & 0.28 & 0.25 & 0.29 \\
$E_{11} / E_{33}$ & 2.93 & 1.95 & 2.19 & 2.05 & 1.75 & 2.70 & 2.65 & 3.75 & 1.99 & 1.93 & 2.36 \\
$\zeta_{1}$ & -0.16 & -0.21 & -0.18 & -0.02 & -0.20 & -0.29 & -0.19 & -0.60 & -0.30 & -0.30 & -0.15 \\
$\zeta_{2}$ & 0.10 & 0.17 & 0.17 & 0.26 & 0.22 & 0.10 & 0.13 & -0.08 & 0.14 & 0.16 & 0.15 \\
\hline
\end{tabular}




\begin{tabular}{cccccc}
\hline$E_{33}$ & 11.3 & 9.7 & 17.2 & 21.0 & 45.4 \\
$E_{11}$ & 31.7 & 25.8 & 32.2 & 33 & 57.8 \\
$\nu_{31}$ & 0.18 & 0.17 & 0.14 & 0.14 & 0.27 \\
$\nu_{12}$ & 0.43 & 0.27 & 0.34 & 0.27 & 0.29 \\
$E_{11} / E_{33}$ & 2.81 & 2.66 & 1.87 & 1.57 & 1.27 \\
$\zeta_{1}$ & -0.34 & -0.24 & -0.07 & 0.04 & -0.25 \\
$\zeta_{2}$ & 0.02 & 0.10 & 0.21 & 0.33 & 0.29 \\
\hline
\end{tabular}

If the volumetric-deviatoric split is adopted, then the elastic parameters on the microplane are calculated from Eqs.(12-17). Substituting Eqs.(20-21) into Eq.(13) and setting the expression in Eq.(13) greater than 0, one finds that the macro-scale elastic parameters should satisfy the condition

$$
\zeta_{2}=\frac{E_{33}}{E_{11}}\left(1-\nu_{12}\right)-\nu_{31}>0
$$

The values of $\zeta_{2}$ are listed in the Table 1. It shows that the microplane elastic parameters are positive except when the degree of anisotropy, $E_{11} / E_{33}>3.75$. So there is no problem for shales, since (Sayers, 2013) showed that their degrees of anisotropy are usually between 1 and 2.75 .

Appendix III: Calculation of microplane elastic moduli by weighting of the spherical microplanes as a function of the microplane dip angle

By weighting of the spherical microplanes (and strength limits) as a function of the latitude angle, one can simulate the full orthotropic material. In this strategy, the microplane elastic moduli become functions of microplane dip angle $\theta$.

In the case that the volumetric-deviatoric split is considered, there are six microplane elastic parameters, $E_{N}^{s}, E_{M}^{s}, E_{L}^{s}$ on the spherical microplanes, and three, $E_{N}^{c}, E_{M}^{s}, E_{L}^{c}$, on the cylindrical microplanes; $E_{N}^{s}, E_{M}^{s}$ and $E_{L}^{s}$ are now functions of spherical microplane dip angle $\theta$ while $E_{N}^{c}$, while $E_{M}^{s}$ and $E_{L}^{c}$ are the same for all cylindrical microplanes. Based on the principle of virtual work, the fourth-order stiffness tensor can be computed by the following equation:

$$
\begin{aligned}
\bar{C}_{i j k l} & =\alpha \frac{3}{2 \pi} \int_{\Omega}\left[E_{N}^{s}(\theta) N_{i j}^{s} N_{k l}^{s}+E_{L}^{s}(\theta) L_{i j}^{s} L_{k l}^{s}+E_{M}^{s}(\theta) M_{i j}^{s} M_{k l}^{s}\right] \mathrm{d} \Omega \\
& +(1-\alpha) \frac{1}{\pi} \int_{S}\left(E_{N}^{c} N_{i j}^{c} N_{k l}^{c}+E_{L}^{c} L_{i j}^{c} L_{k l}^{c}+E_{M}^{c} M_{i j}^{c} M_{k l}^{c}\right) \mathrm{d} S
\end{aligned}
$$

According to the (Salviato et al., 2016), Eq.(75) can also be written as:

$$
\bar{C}_{i j k l}=\alpha \frac{3}{2 \pi} \int_{\Omega} P^{T} E P d \Omega+(1-\alpha) \frac{1}{\pi} \int_{S}\left(E_{N}^{c} N_{i j}^{c} N_{k l}^{c}+E_{L}^{c} L_{i j}^{c} L_{k l}^{c}+E_{M}^{c} M_{i j}^{c} M_{k l}^{c}\right) \mathrm{d} S
$$

where

$$
P=\left[\begin{array}{cccccc}
N_{11}^{s} & N_{22}^{s} & N_{33}^{s} & \sqrt{2} N_{23}^{s} & \sqrt{2} N_{31}^{s} & \sqrt{2} N_{12}^{s} \\
M_{11}^{s} & M_{22}^{s} & M_{33}^{s} & \sqrt{2} M_{23}^{s} & \sqrt{2} M_{31}^{s} & \sqrt{2} M_{12}^{s} \\
L_{11}^{s} & L_{22}^{s} & L_{33}^{s} & \sqrt{2} L_{23}^{s} & \sqrt{2} L_{31}^{s} & \sqrt{2} L_{12}^{s}
\end{array}\right]
$$

and

$$
E=\left[\begin{array}{ccc}
E_{N}^{s}(\theta) & 0 & 0 \\
0 & E_{M}^{s}(\theta) & 0 \\
0 & 0 & E_{L}^{s}(\theta)
\end{array}\right]
$$


The variation of $E_{N}^{s}, E_{M}^{s}$ and $E_{L}^{s}$ may be defined as:

$$
\begin{aligned}
E_{N}^{s} & =\left(a_{1} \cos ^{2} \theta+a_{2} \sin ^{2} \theta\right)^{-1} \\
E_{M}^{s} & =\left(a_{3} \cos ^{2} \theta+a_{4} \sin ^{2} \theta\right)^{-1} \\
E_{L}^{s} & =\left(a_{5} \cos ^{2} \theta+a_{6} \sin ^{2} \theta\right)^{-1}
\end{aligned}
$$

Substituting Eqs.(77-81) into Eq.(76) and using the same method as in Appendix I, one can get the expressions for the microplane elastic modulus.

Appendix IV: Calculation of microplane elastic moduli of the orthotropic microplane configuration

One possible microplane configuration for orthotropic materials, which have 9 independent elastic parameters, may involve two cylindrical microplane models with orthogonal axes, as diagrammed in Fig.4. Based on the microplane theory, the macroscopic continuum stress tensor in this microplane configuration can be obtained

$$
\begin{aligned}
\sigma_{i j}= & \alpha \frac{3}{2 \pi} \int_{\Omega}\left(\sigma_{N}^{s} N_{i j}^{s}+\sigma_{L}^{s} L_{i j}^{s}+\sigma_{M}^{s} M_{i j}^{s}\right) d \Omega+\gamma \frac{1}{\pi} \int_{S_{1}}\left(\sigma_{N}^{c} N_{i j}^{c}+\sigma_{L}^{c} L_{i j}^{c}+\sigma_{M}^{c} M_{i j}^{c}\right) \mathrm{d} S_{1} \\
& +(1-\alpha-\gamma) \frac{1}{\pi} \int_{S_{2}}\left(\sigma_{N}^{v} N_{i j}^{v}+\sigma_{L}^{v} L_{i j}^{v}+\sigma_{M}^{v} M_{i j}^{v}\right) \mathrm{d} S_{2}
\end{aligned}
$$

Here the superscript $v$ labels the variables on the vertical cylindrical microplane; $\alpha$ and $\gamma$ are two empirical factors less than 1; other notations have the same meaning as described in Eqs.(3-7)

Similar to the spherocylindrical microplane model Eqs.(9-10), the elastic behavior on the vertical cylindrical microplane is described as

$$
\sigma_{N}^{v}=E_{N}^{v} \epsilon_{N}^{v}, \quad \sigma_{L}^{v}=E_{L}^{v} \epsilon_{L}^{v}, \quad \sigma_{M}^{v}=E_{M}^{v} \epsilon_{M}^{v}
$$

Here $E_{N}^{v}, E_{L}^{v}$ and $E_{M}^{v}$ are the elastic parameters on the vertical cylindrical microplane, considered as independent of microplane orientation. Substitution of Eqs.(9-10) and Eq.(83) into Eq.(82) leads to the fourth-order stiffness tensor:

$$
\begin{aligned}
\bar{C}_{i j k l}= & \alpha \frac{3}{2 \pi} \int_{\Omega}\left(E_{D}^{s} N_{i j}^{s} N_{k l}^{s}+\frac{1}{3}\left(E_{V}-E_{D}^{s}\right) N_{i j}^{s} \delta_{k l}+E_{L}^{s} L_{i j}^{s} L_{k l}^{s}+E_{M}^{s} M_{i j}^{s} M_{k l}^{s}\right) \mathrm{d} \Omega \\
& +\gamma \frac{1}{\pi} \int_{S_{1}}\left(E_{N}^{c} N_{i j}^{c} N_{k l}^{c}+E_{L}^{c} L_{i j}^{c} L_{k l}^{c}+E_{M}^{c} M_{i j}^{c} M_{k l}^{c}\right) \mathrm{d} S_{1} \\
& +(1-\alpha-\gamma) \frac{1}{\pi} \int_{S_{2}}\left(E_{N}^{v} N_{i j}^{v} N_{k l}^{v}+E_{L}^{v} L_{i j}^{v} L_{k l}^{v}+E_{M}^{v} M_{i j}^{v} M_{k l}^{v}\right) \mathrm{d} S_{2}
\end{aligned}
$$

The same method as introduced in Appendix I now yields:

$$
\int_{S_{2}} \bar{N}_{I}^{v} \bar{N}_{J}^{v} d S_{2}=\frac{1}{N_{m}^{v}} \sum_{\mu=1}^{N_{m}^{v}} \bar{N}_{I}^{v} \bar{N}_{J}^{v}=\pi\left[\begin{array}{cccccc}
0 & 0 & 0 & 0 & 0 & 0 \\
0 & 0.375 & 0.125 & 0 & 0 & 0 \\
0 & 0.125 & 0.375 & 0 & 0 & 0 \\
0 & 0 & 0 & 0 & 0 & 0 \\
0 & 0 & 0 & 0 & 0.125 & 0 \\
0 & 0 & 0 & 0 & 0 & 0
\end{array}\right]
$$




$$
\begin{gathered}
\int_{S_{2}} \bar{L}_{I}^{v} \bar{L}_{J}^{v} d S_{2}=\frac{1}{N_{m}^{v}} \sum_{\mu=1}^{N_{m}^{v}} \bar{L}_{I}^{v} \bar{L}_{J}^{v}=\pi\left[\begin{array}{cccccc}
0 & 0 & 0 & 0 & 0 & 0 \\
0 & 0.125 & -0.125 & 0 & 0 & 0 \\
0 & -0.125 & 0.125 & 0 & 0 & 0 \\
0 & 0 & 0 & 0 & 0 & 0 \\
0 & 0 & 0 & 0 & 0.125 & 0 \\
0 & 0 & 0 & 0 & 0 & 0
\end{array}\right] \\
\int_{S_{2}} \bar{M}_{I}^{v} \bar{M}_{J}^{v} d S_{2}=\frac{1}{N_{m}^{v}} \sum_{\mu=1}^{N_{m}^{v}} \bar{M}_{I}^{v} \bar{M}_{J}^{v}=\pi\left[\begin{array}{ccccccc}
0 & 0 & 0 & 0 & 0 & 0 \\
0 & 0 & 0 & 0 & 0 & 0 \\
0 & 0 & 0 & 0 & 0 & 0 \\
0 & 0 & 0 & 0.125 & 0 & 0 \\
0 & 0 & 0 & 0 & 0 & 0 \\
0 & 0 & 0 & 0 & 0 & 0.125
\end{array}\right]
\end{gathered}
$$

Similar to the procedure in Appendix I, comparison of the left and right sides of Eq.(84) gives the elastic parameters on the microplane:

$$
\begin{aligned}
& E_{V}=\left(C_{11}+C_{33}-C_{22}+2 C_{13}\right) / \alpha \\
& E_{L}^{s}=E_{M}^{s}=E_{T}^{s}=\left(C_{11}+C_{33}-C_{22}-C_{13}\right) / \alpha \\
& E_{D}^{s}=\left(C_{11}+C_{33}-C_{22}-C_{13}\right) / \alpha \\
& E_{N}^{c}=2\left(C_{22}-C_{33}+C_{12}-C_{13}\right) / \gamma \\
& E_{L}^{c}=2\left(C_{22}-C_{33}-3 C_{12}+3 C_{13}\right) / \gamma \\
& E_{M}^{c}=\left[4\left(C_{22}-C_{33}+C_{13}-C_{12}\right)-8 C_{66}+8 C_{55}\right] / \gamma \\
& E_{N}^{v}=2\left(C_{22}-C_{11}+C_{23}-C_{13}\right) /(1-\alpha-\gamma) \\
& E_{L}^{v}=2\left(C_{22}-C_{11}-3 C_{23}+3 C_{13}\right) /(1-\alpha-\gamma) \\
& E_{M}^{v}=\left[4\left(C_{22}-C_{11}-C_{23}+C_{13}\right)-8 C_{44}+8 C_{55}\right] /(1-\alpha-\gamma)
\end{aligned}
$$

When $\alpha+\gamma=1$, the foregoing equations can be used to compute the microplane elastic moduli of the spherocylindrical microplane model. When $\alpha=1$, the foregoing equations can be used to compute microplane elastic moduli of the general microplane model.

Acknowledgement: Partial financial support from the U.S. Department of Energy through subcontract No. 37008 of Northwestern University with Los Alamos National Laboratory is gratefully acknowledged. The simulation of fracturing damage received also some support from ARO grant W911NF-15-101240 to Northwestern University. The first author wishes to thank Department of Science and Technology of Sichuan Province (No.2015JY0280, No.2012FZ0124), NSFC (No.41472271) and CSC for supporting him as a Research Fellow at Northwestern University.

\section{References}

Akono, A.-T., 2016. Energetic size effect law at the microscopic scale: Application to progressive-load scratch testing. Journal of Nanomechanics and Micromechanics 6 (2), 04016001.

Asaro, R. J., Rice, J. R., 1977. Strain localization in ductile single crystals. Journal of the Mechanics and Physics of Solids 25 (5), 309-338. 
Batdorf, S. B., Budiansky, B., 1949. A mathematical theory of plasticity based on the concept of slip. In: Technical Note No. 1871. Nat. Advisory Committee for Aeronautics, Washington,D.C.

Bažant, Z. P., 1976. Instability, ductility, and size effect in strain-softening concrete. Journal of the Engineering Mechanics Division 102 (2), 331-344.

Bažant, Z. P., 1984. Microplane model for strain-controlled inelastic behaviour. In: Chapter 3 in Mechanics of Engineering Materials,45-59. ed. by C. S. Desai and R.H. Gallagher, J. Wiley, London.

Bažant, Z. P., 2017. Simulation of hydraulic fracture in shale: Crack branching and spherocylindrical orthotropic microplane constitutive model. In: Proc., 14th Int. Congress of Fracture (honoring G.I. Barenblatt at his 90th birthday). Rhodes (based on privately communicated note to C. Li and F. Caner, Jan. 15, 2016).

Bažant, Z. P., Adley, M. D., Carol, I., Jirásek, M., Akers, S. A., Rohani, B., Cargile, J. D., Caner, F. C., 2000a. Large-strain generalization of microplane model for concrete and application. Journal of Engineering Mechanics 126 (9), 971-980.

Bažant, Z. P., Caner, F. C., 2005. Microplane model m5 with kinematic and static constraints for concrete fracture and anelasticity. i: Theory. Journal of Engineering Mechanics 131 (1), $31-40$.

Bažant, Z. P., Caner, F. C., 2014. Impact comminution of solids due to local kinetic energy of high shear strain rate: I. continuum theory and turbulence analogy. Journal of the Mechanics and Physics of Solids 64, 223-235.

Bažant, Z. P., Caner, F. C., Carol, I., Adley, M. D., Akers, S. A., 2000b. Microplane model m4 for concrete. i: Formulation with work-conjugate deviatoric stress. Journal of Engineering Mechanics 126 (9), 944-953.

Bažant, Z. P., Gambarova, P. G., 1984. Crack shear in concrete: Crack band microflane model. Journal of Structural Engineering 110 (9), 2015-2035.

Bažant, Z. P., Jirásek, M., Hubler, M., Carol, I., 2015. Rilem draft recommendation: Tc-242mdc multi-decade creep and shrinkage of concrete: material model and structural analysis. model b4 for creep, drying shrinkage and autogenous shrinkage of normal and high-strength concretes with multi-decade applicability. Materials and structures 48 (4), 753-770.

Bažant, Z. P., Kim, J.-K., 1986. Creep of anisotropic clay: microplane model. Journal of Geotechnical Engineering 112 (4), 458-475.

Bažant, Z. P., Oh, B., 1986. Efficient numerical integration on the surface of a sphere. ZAMMJournal of Applied Mathematics and Mechanics/Zeitschrift für Angewandte Mathematik und Mechanik 66 (1), 37-49.

Bažant, Z. P., Oh, B. H., 1983. Crack band theory for fracture of concrete. Materials and structures $16(3), 155-177$.

Bažant, Z. P., Oh, B. H., 1985. Microplane model for progressive fracture of concrete and rock. Journal of Engineering Mechanics 111 (4), 559-582. 
Bažant, Z. P., Prat, P. C., 1987. Creep of anisotropic clay: new microplane model. Journal of Engineering Mechanics 113 (7), 1050-1064.

Bažant, Z. P., Prat, P. C., 1988. Microplane model for brittle-plastic material: I. theory. Journal of Engineering Mechanics 114 (10), 1672-1688.

Bažant, Z. P., Salviato, M., Chau, V. T., Viswanathan, H., Zubelewicz, A., 2014. Why fracking works. Journal of Applied Mechanics 81 (10), 101010-101010-10.

Bažant, Z. P., Xiang, Y., Prat, P. C., 1996. Microplane model for concrete. i: stress-strain boundaries and finite strain. Journal of Engineering Mechanics 122 (3), 245-254.

Bažant, Z. P., Zi, G., 2003. Microplane constitutive model for porous isotropic rocks. International Journal for Numerical and Analytical Methods in Geomechanics 27 (1), 25-47.

Brocca, M., Bažant, Z. P., Daniel, I. M., 2001. Microplane model for stiff foams and finite element analysis of sandwich failure by core indentation. International Journal of Solids and Structures 38 (4445), 8111-8132.

Caner, F. C., Bažant, Z. P., 2000. Microplane model m4 for concrete. ii: Algorithm and calibration. Journal of Engineering Mechanics 126 (9), 954-961.

Caner, F. C., Bažant, Z. P., 2011. Microplane model m6f for fiber reinforced concrete. Proc., in: XI Int. Conf. on Computational Plasticity Fundamentals and Applications, COMPLAS 2011, Barcelona, Spain, pp. 796807.

Caner, F. C., Bažant, Z. P., 2013. Microplane model m7 for plain concrete. i: Formulation. Journal of Engineering Mechanics 139 (12), 1714-1723.

Caner, F. C., Bažant, Z. P., 2014. Impact comminution of solids due to local kinetic energy of high shear strain rate: Ii-microplane model and verification. Journal of the Mechanics and Physics of Solids 64, 236-248.

Carol, I., Bažant, Z. P., Prat, P. C., 1991. Geometric damage tensor based on microplane model. Journal of Engineering Mechanics 117 (10), 2429-2448.

Chang, C., Zoback, M. D., 2009. Viscous creep in room-dried unconsolidated gulf of mexico shale (i): Experimental results. Journal of Petroleum Science and Engineering 69 (3), 239246.

Chen, L., Shao, J. F., Huang, H. W., 2010. Coupled elastoplastic damage modeling of anisotropic rocks. Computers and Geotechnics 37 (1), 187-194.

Chen, X., Bažant, Z. P., 2014. Microplane damage model for jointed rock masses. International Journal for Numerical and Analytical Methods in Geomechanics 38 (14), 1431-1452.

Clark, C., Burnham, A., Harto, C., Horner, R., 2013. Hydraulic fracturing and shale gas production: technology, impacts, and policy. Tech. Rep. ANL/EVS/R-12/5, Argonne National Lab.

Cusatis, G., Beghini, A., Bažant, Z. P., 2008. Spectral stiffness microplane model for quasibrittle composite laminatespart i: Theory. Journal of Applied Mechanics 75 (2), 021009-021009-9. 
Duveau, G., Shao, J., Henry, J., 1998. Assessment of some failure criteria for strongly anisotropic geomaterials. Mechanics of Cohesive-frictional Materials 3 (1), 1-26.

Gao, Z., Zhao, J., Yao, Y., 2010. A generalized anisotropic failure criterion for geomaterials. International Journal of Solids and Structures 47 (22), 3166-3185.

Gautam, R., Wong, R. C., 2006. Transversely isotropic stiffness parameters and their measurement in colorado shale. Canadian Geotechnical Journal 43 (12), 1290-1305.

Hahn, H. T., Tsai, S. W., 1980. Introduction to composite materials, Chapter 9: micromechnics. CRC Press.

Heng, S., Guo, Y., Yang, C., Daemen, J. J., Li, Z., 2015. Experimental and theoretical study of the anisotropic properties of shale. International Journal of Rock Mechanics and Mining Sciences 74, 58-68.

Hill, R., 1998. The mathematical theory of plasticity. Vol. 11. Oxford university press.

Hoover, C. G., Bažant, Z. P., 2014. Cohesive crack, size effect, crack band and work-of-fracture models compared to comprehensive concrete fracture tests. International Journal of Fracture 187 (1), 133-143.

Hull, K. L., Abousleiman, Y. N., Han, Y., Al-Muntasheri, G. A., Hosemann, P., Parker, S. S., Howard, C. B., 2017. Nanomechanical characterization of the tensile modulus of rupture for kerogen-rich shale. SPE Journal.

Jaeger, J., 1960. Shear failure of anistropic rocks. Geological Magazine 97 (1), 65-72.

Jin, C., Salviato, M., Li, W., Cusatis, G., 2017a. Elastic microplane formulation for transversely isotropic materials. Journal of Applied Mechanics 84 (1), 011001-011001-14.

Jin, W., Xu, H., Arson, C., Busetti, S., 2017b. Computational model coupling mode ii discrete fracture propagation with continuum damage zone evolution. International Journal for Numerical and Analytical Methods in Geomechanics 41 (2), 223-250.

Jones, R. M., 1975. Mechanics of composite materials. Vol. 193. Scripta Book Company Washington, DC.

Kim, H., Cho, J.-W., Song, I., Min, K.-B., 2012. Anisotropy of elastic moduli, p-wave velocities, and thermal conductivities of asan gneiss, boryeong shale, and yeoncheon schist in korea. Engineering Geology 147, 68-77.

Kirane, K., Salviato, M., Bažant, Z. P., 2015. Microplane triad model for simple and accurate prediction of orthotropic elastic constants of woven fabric composites. Journal of Composite Materials 50 (9), 1247-1260.

Kirane, K., Salviato, M., Bažant, Z. P., 2016. Microplane-triad model for elastic and fracturing behavior of woven composites. Journal of Applied Mechanics 83 (4), 041006-041006-14.

Lee, Y.-K., Pietruszczak, S., 2015. Tensile failure criterion for transversely isotropic rocks. International Journal of Rock Mechanics and Mining Sciences 79, 205-215. 
Levasseur, S., Welemane, H., Kondo, D., 2015. A microcracks-induced damage model for initially anisotropic rocks accounting for microcracks closure. International Journal of Rock Mechanics and Mining Sciences 77, 122-132.

Li, W., Rezakhani, R., Jin, C., Zhou, X., Cusatis, G., 2017. A multiscale framework for the simulation of the anisotropic mechanical behavior of shale. International Journal for Numerical and Analytical Methods in Geomechanics, online.

Masri, M., Sibai, M., Shao, J.-F., Mainguy, M., 2014. Experimental investigation of the effect of temperature on the mechanical behavior of tournemire shale. International Journal of Rock Mechanics and Mining Sciences 70, 185-191.

McDowell, D. L., 2008. Viscoplasticity of heterogeneous metallic materials. Materials Science and Engineering: R: Reports 62 (3), 67-123.

Mohamadi, M., Wan, R. G., 2016. Strength and post-peak response of colorado shale at high pressure and temperature. International Journal of Rock Mechanics and Mining Sciences 84, $34-46$.

Mokhtari, M., Bui, B. T., Tutuncu, A. N., 2014. Tensile failure of shales: impacts of layering and natural fractures. In: SPE Western North American and Rocky Mountain Joint Meeting. Society of Petroleum Engineers.

Niandou, H., Shao, J., Henry, J., Fourmaintraux, D., 1997. Laboratory investigation of the mechanical behaviour of tournemire shale. International Journal of Rock Mechanics and Mining Sciences 34 (1), 3-16.

Pietruszczak, S., Lydzba, D., Shao, J.-F., 2002. Modelling of inherent anisotropy in sedimentary rocks. International Journal of Solids and Structures 39 (3), 637-648.

Ramamurthy, T., Rao, G., Singh, J., 1988. A strength criterion for anisotropic rocks. In: Fifth Australia-New Zealand Conference on Geomechanics: Prediction Versus Performance. Barton, ACT: Institution of Engineers, Australia.

Salviato, M., Ashari, S. E., Cusatis, G., 2016. Spectral stiffness microplane model for damage and fracture of textile composites. Composite Structures 137, 170-184.

Sayers, C. M., 2013. The effect of anisotropy on the young's moduli and poisson's ratios of shales. Geophysical Prospecting 61 (2), 416-426.

Šmilauer, V., Hoover, C. G., Bažant, Z. P., Caner, F. C., Waas, A. M., Shahwan, K. W., 2011. Multiscale simulation of fracture of braided composites via repetitive unit cells. Engineering Fracture Mechanics 78 (6), 901-918.

Sone, H., 2012. Mechanical properties of shale gas reservoir rocks, and its relation to the in-situ stress variation observed in shale gas reservoirs. Ph.D. thesis, Stanford University.

Taylor, G., 1938. The plastic strain of metals. Journal Inst Metals 62, 307-324.

Tsai, S. W., Wu, E. M., 1971. A general theory of strength for anisotropic materials. Journal of Composite Materials 5 (1), 58-80. 
Vervoort, A., Min, K.-B., Konietzky, H., Cho, J.-W., Debecker, B., Dinh, Q.-D., Frühwirt, T., Tavallali, A., 2014. Failure of transversely isotropic rock under brazilian test conditions. International Journal of Rock Mechanics and Mining Sciences 70, 343-352.

Waters, G. A., Lewis, R. E., Bentley, D., 2011. The effect of mechanical properties anisotropy in the generation of hydraulic fractures in organic shales. In: SPE Annual Technical Conference and Exhibition. Society of Petroleum Engineers.

Xie, H., Gao, F., Ju, Y., Xie, L., Yang, Y., Wang, J., 2016. Novel idea of the theory and application of $3 \mathrm{~d}$ volume fracturing for stimulation of shale gas reservoirs. Chinese Science Bulletin 61 (1), 36-46. 

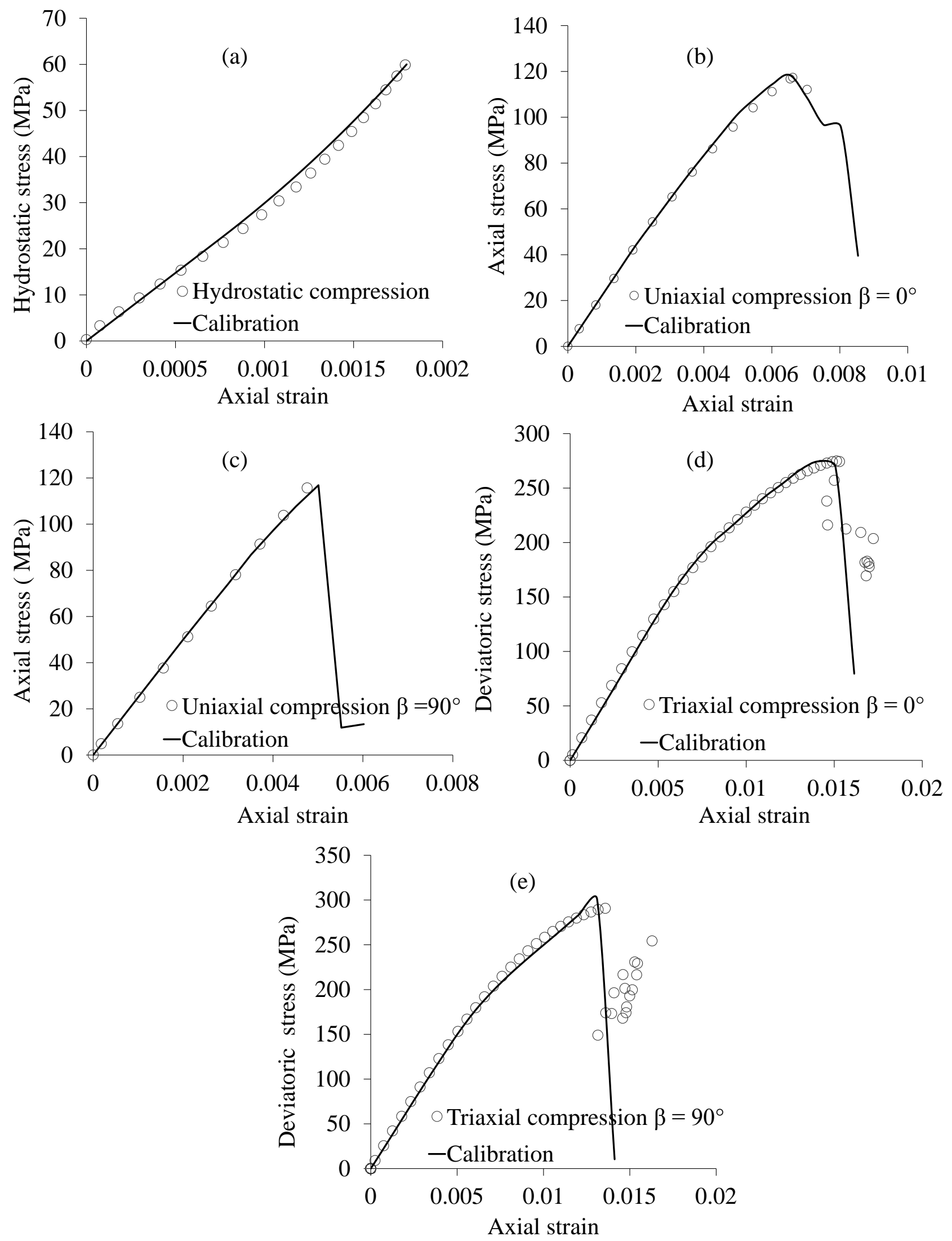

Figure 10: Results of calibration of the present model: (a) hydrostatic compression for $\beta=0^{\circ}$ (b) uniaxial compression for $\beta=0^{\circ}$; (c) uniaxial compression for $\beta=90^{\circ}$; (d) triaxial compression for $\beta=0^{\circ}$; triaxial compression for $\beta=0^{\circ}$. 


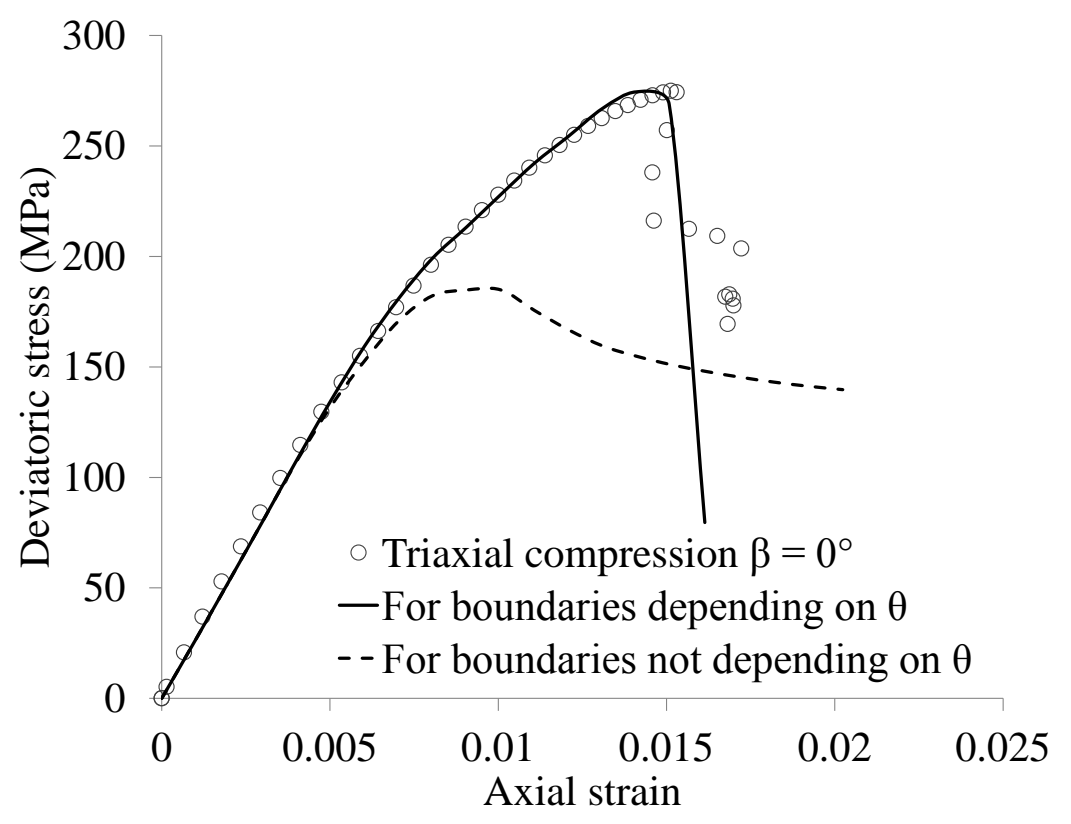

Figure 11: The necessity of making the boundary magnitude of Longmaxi shale dependent on microplane dip angle $\theta$ (taking $0-60$ as an example). 

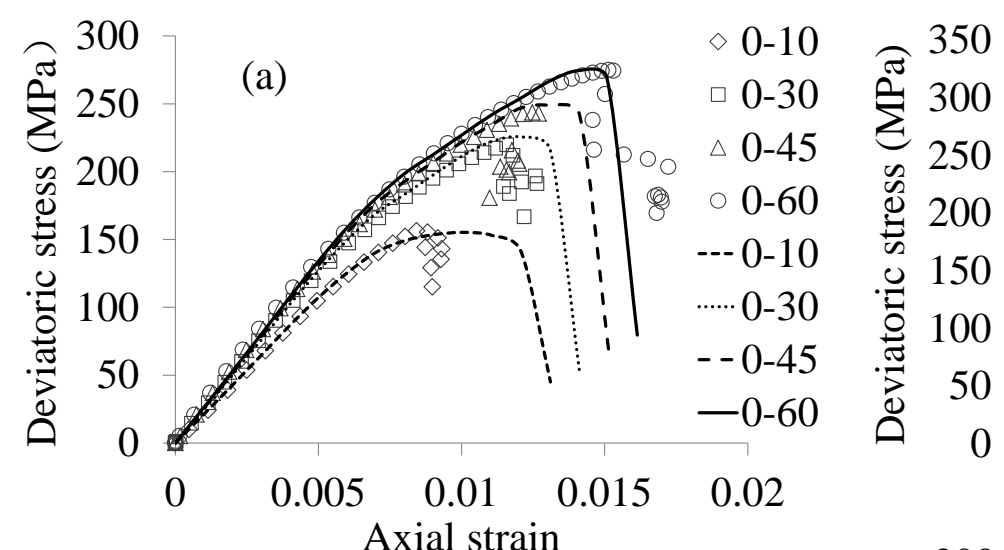

(b)

$---15-10$

... $15-30$

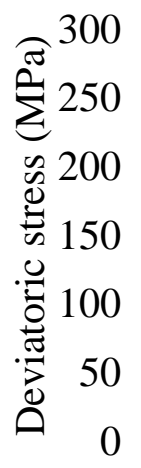

$---30-10$

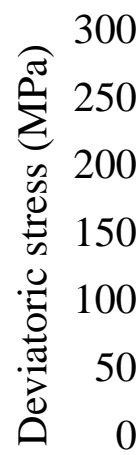

$\begin{array}{lllll}0 & 0.005 & 0.01 & 0.015 & 0.02\end{array}$

Axial strain

--45-10

…. 30-30

- - 30-45

-30-60

$\diamond 30-10$

- 30-30

$\triangle 30-45$

- 30-60

$\begin{array}{lllll}0 & 0.005 & 0.01 & 0.015 & 0.02\end{array}$
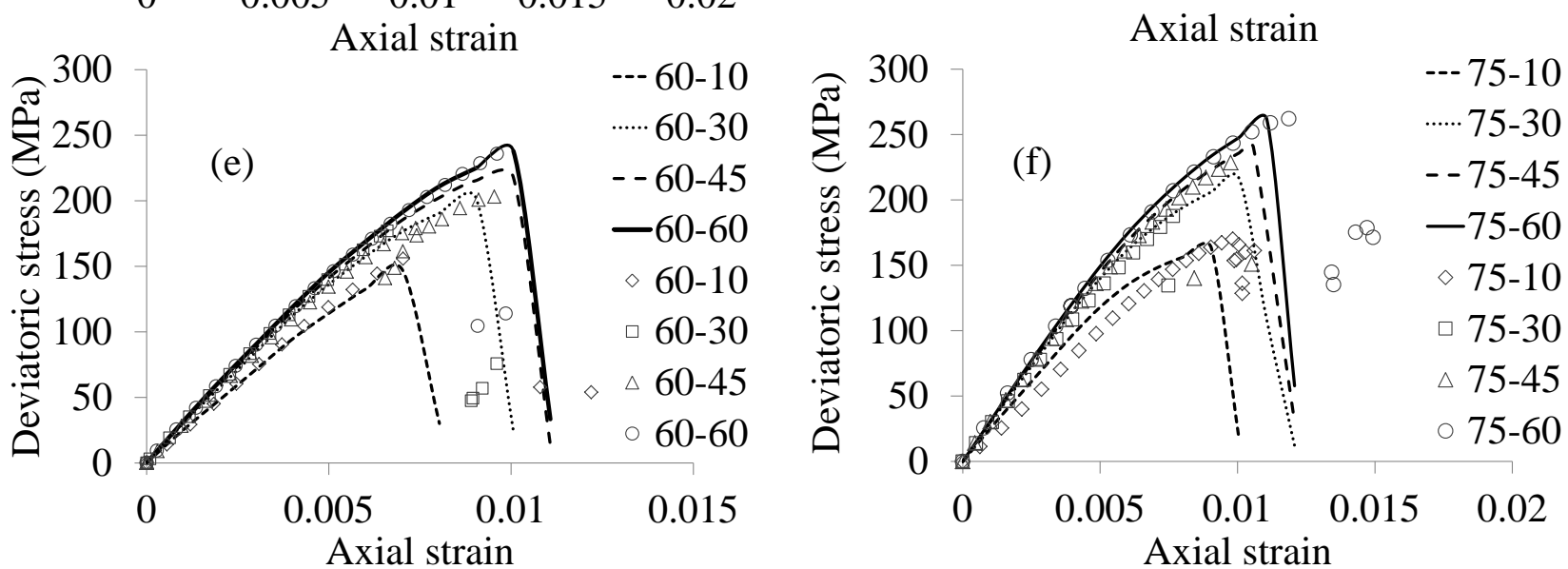

Axial strain
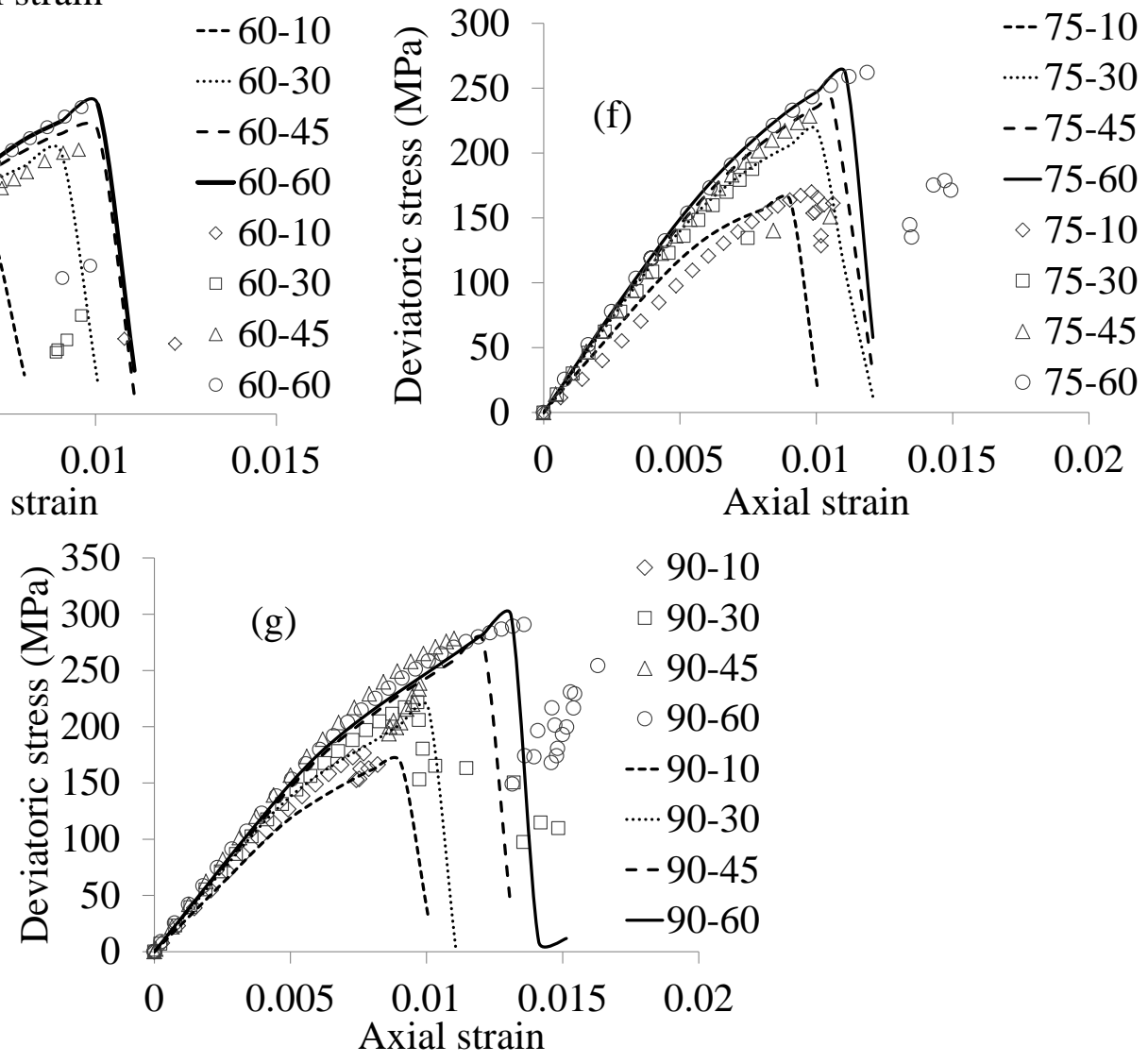

Figure 12: Numerical simulations of triaxial tests of Longmaxi shale under different confinements and different bedding layer orientations: (a) $\beta=0^{\circ}$; (b) $\beta=15^{\circ}$; (c) $\beta=30^{\circ}$; (d) $\beta=45^{\circ}$; $\beta=60^{\circ}$; (f) $\beta=75^{\circ}$; (g) $\beta=90^{\circ}$. 

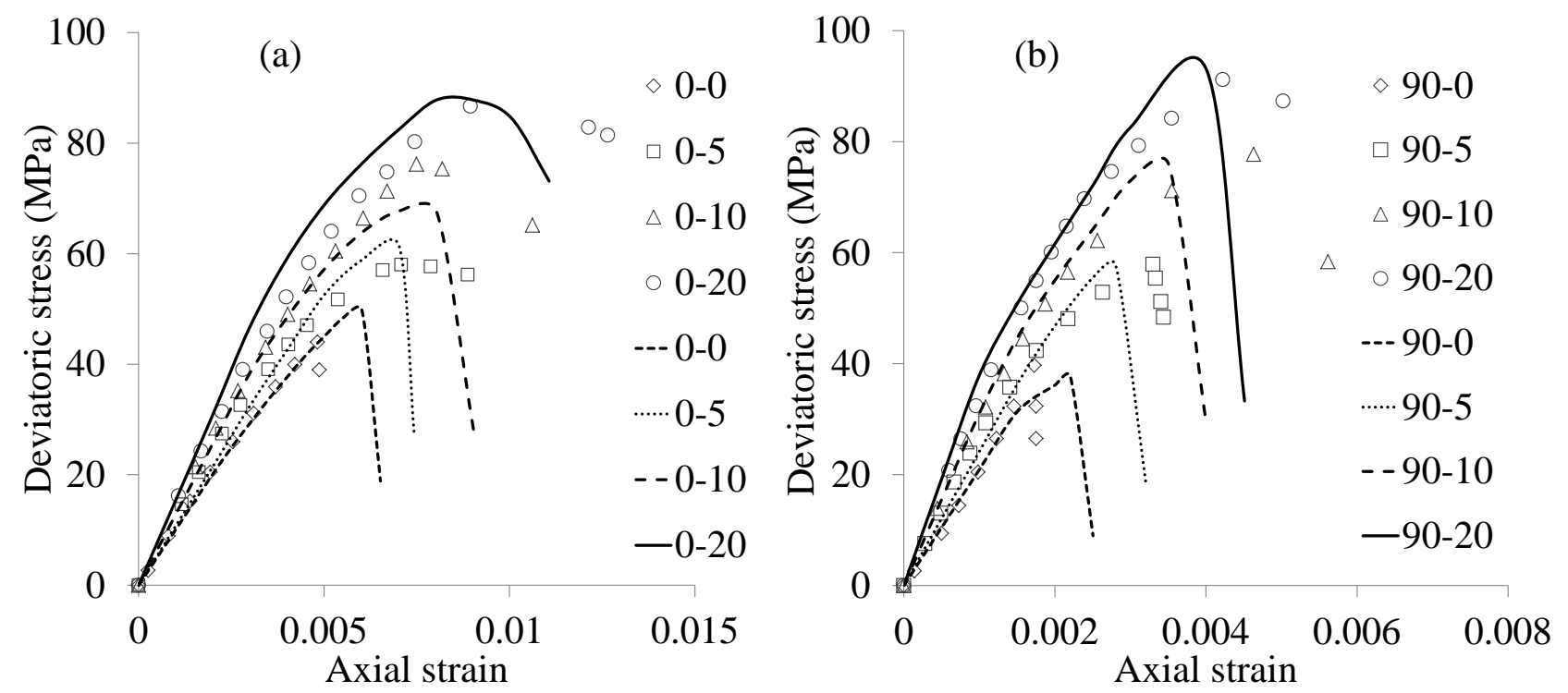

Figure 13: Simulation results of Tournemire shale: (a) uniaxial and triaxial compression tests for $\beta=0^{\circ}$; (b) uniaxial and triaxial compression tests for $\beta=90^{\circ}$. 